Curtis Madsen ${ }^{1}$ / Angel Goni Moreno ${ }^{2}$ / Zachary Palchick ${ }^{3}$ / Umesh P4 / Nicholas Roehner ${ }^{5}$ / Bryan Bartley ${ }^{5}$ / Swapnil Bhatia ${ }^{1}$ Shyam Bhakta ${ }^{6}$ / Mike Bissell ${ }^{7}$ / Kevin Clancy ${ }^{8}$ / Robert Sidney Cox ${ }^{9}$ / Thomas Gorochowski ${ }^{10}$ / Raik Grunberg ${ }^{11}$ / Augustin Luna ${ }^{12}$ / James McLaughlin ${ }^{2}$ / Tramy Nguyen ${ }^{13}$ / Nicolas Le Novere ${ }^{14}$ / Matthew Pocock ${ }^{15}$ / Herbert Sauro ${ }^{16}$ / James Scott-Brown ${ }^{17}$ / John T. Sexton ${ }^{6}$ / Guy-Bart Stan ${ }^{17}$ / Jeffrey J. Tabor ${ }^{6} /$ Christopher A. Voigt $^{18} /$ Zach Zundel $^{13} /$ Chris Myers $^{13}$ / Jacob Beal ${ }^{5}$ / Anil Wipat ${ }^{2}$

\title{
Synthetic Biology Open Language Visual (SBOL Visual) Version 2.1
}

\author{
${ }^{1}$ Boston University, Boston, MA, USA \\ ${ }^{2}$ Newcastle University, Newcastle, UK \\ ${ }^{3}$ Zymergen, Emeryville, CA, USA \\ ${ }^{4}$ Kerala Technological University, Thiruvananthapuram, India \\ ${ }^{5}$ Raytheon BBN Technologies, Cambridge, MA, USA, E-mail: jakebeal@ieee.org. https://orcid.org/0000-0002-1663-5102. \\ ${ }^{6}$ Rice University, Houston, TX, USA \\ ${ }^{7}$ Amyris, Inc., Emeryville, CA, USA \\ ${ }^{8}$ Thermo Fisher Scientific, San Diego, CA, USA \\ ${ }^{9}$ Kobe University, Kobe, Japan \\ ${ }^{10}$ University of Bristol, Bristol, UK \\ ${ }^{11}$ KAUST, Thuwal, Saudi Arabia \\ ${ }^{12}$ Harvard Medical School, Boston, MA, USA \\ 13 University of Utah, Salt Late City, UT, USA \\ 14 Babraham Institute, Cambridge, UK \\ 15 Turing Ate My Hamster, Ltd., Newcastle, UK \\ ${ }^{16}$ University of Washington, Seattle, WA, USA \\ 17 Imperial College, London, UK \\ ${ }^{18}$ MIT, Cambridge, MA, USA
}

\section{Abstract:}

People who are engineering biological organisms often find it useful to communicate in diagrams, both about the structure of the nucleic acid sequences that they are engineering and about the functional relationships between sequence features and other molecular species. Some typical practices and conventions have begun to emerge for such diagrams. The Synthetic Biology Open Language Visual (SBOL Visual) has been developed as a standard for organizing and systematizing such conventions in order to produce a coherent language for expressing the structure and function of genetic designs. This document details version 2.1 of SBOL Visual, which builds on the prior SBOL Visual 2.0 standard by expanding diagram syntax to include methods for showing modular structure and mappings between elements of a system, interactions arrows that can split or join (with the glyph at the split or join indicating either superposition or a chemical process), and adding new glyphs for indicating genomic context (e.g., integration into a plasmid or genome) and for stop codons.

Keywords: SBOL Visual, Standards, Diagrams

DOI: 10.1515/jib-2018-0101

Received: December 21, 2018; Accepted: May 20, 2019

Article Note: This document does not contain technology or technical data controlled under either the U.S. International Traffic in Arms Regulations or the U.S. Export Administration Regulations. 


\section{Synthetic Biology Open Language Visual (SBOL Visual) Version 2.1}

\author{
Curtis Madsen \\ Angel Goni Moreno \\ Zachary Palchick \\ Umesh P \\ Nicholas Roehner
}

\section{Editors:}

Boston University, USA

Newcastle University

Zymergen, USA

Kerala Technological University, India Raytheon BBN Technologies, USA Chair:

Anil Wipat Newcastle University, UK

editors@sbolstandard.org

Additional authors, by last name:

Bryan Bartley

Raytheon BBN Technologies, USA

Jacob Beal

Swapnil Bhatia

Shyam Bhakta

Mike Bissell

Kevin Clancy

Robert Sidney Cox

Thomas Gorochowski

Raik Grunberg

Augustin Luna

James McLaughlin

Chris Myers

Tramy Nguyen

Nicolas Le Novere

Matthew Pocock

Herbert Sauro

James Scott-Brown

John T. Sexton

Guy-Bart Stan

Jeffrey J. Tabor

Chris Voigt

Zach Zundel
Raytheon BBN Technologies, USA

Boston University, USA

Rice University, USA Amyris, Inc., USA

Thermo Fisher Scientific, USA

Kobe University, Japan

University of Bristol, UK

KAUST, Saudi Arabia

Harvard Medical School, USA

Newcastle University, UK

University of Utah, USA

University of Utah, USA

Babraham Institute, UK

Turing Ate My Hamster, Ltd., UK

University of Washington, USA

Imperial College, UK

Rice University, USA

Imperial College, UK

Rice University, USA

MIT, USA

University of Utah, USA

Version 2.1

November 30, 2018

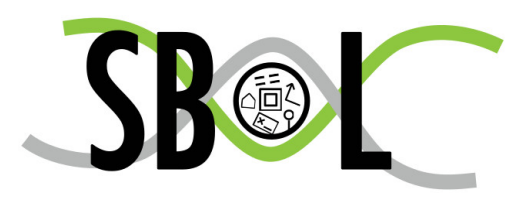




\section{Contents}

1 Purpose 3

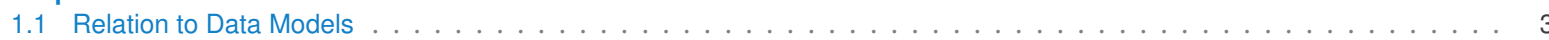

2 Relation to other BBF RFCs and other Standards 3

3 Copyright and License Statement 3

4 SBOL Specification Vocabulary 4

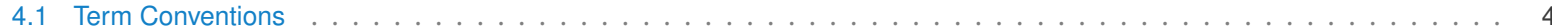

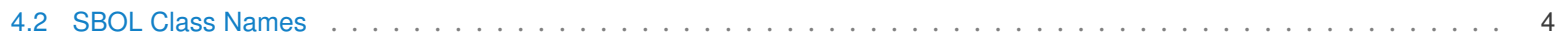

5 SBOL Glyphs

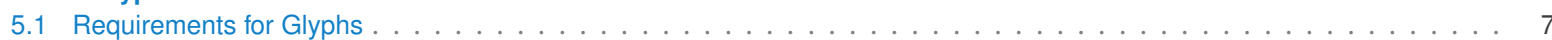

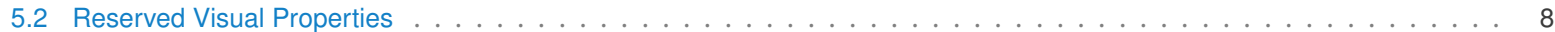

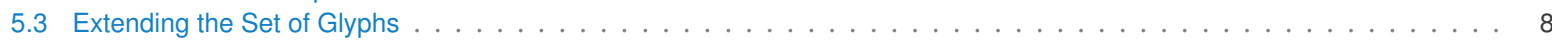

6 SBOL Visual Diagram Language 9

6.1 Nucleic Acid Backbone . . . . . . . . . . . . . . . . . . . . . . . . . . . . . . . . . . . . . . . . .

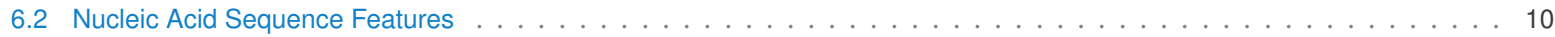

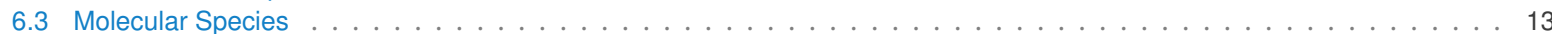

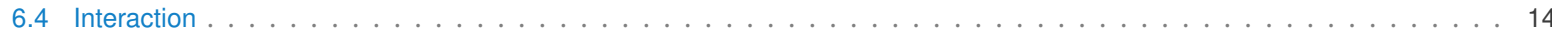

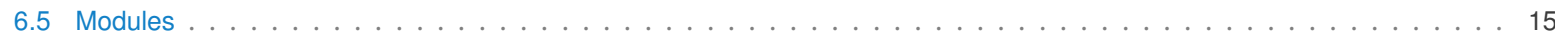

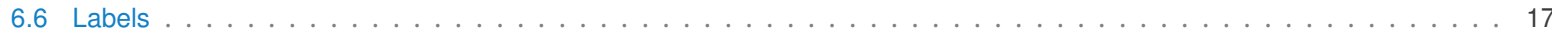

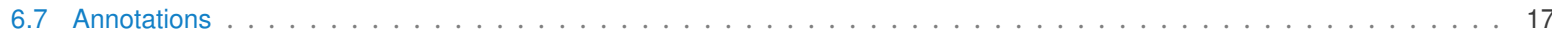

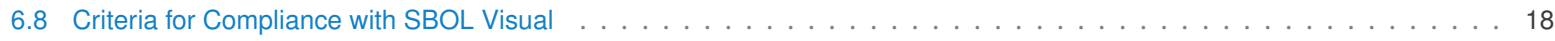

A SBOL Visual Glyphs 19

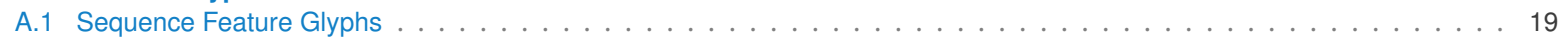

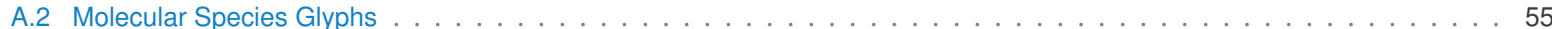

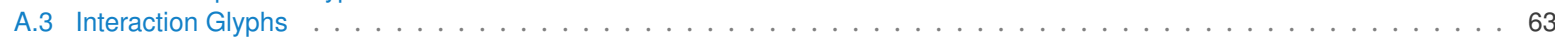

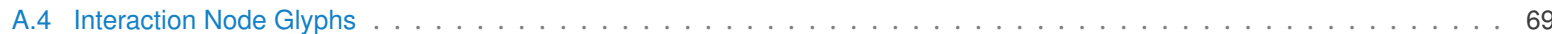

B Examples 73

C Relationship to SBOL Visual 1.0

References 77 


\section{Purpose}

People who engineer biological organisms often find it useful to communicate in diagrams, both about the structure of the nucleic acid sequences that they are engineering and about the functional relationships between sequence features and other molecular species. Some typical practices and conventions have begun to emerge for such diagrams. SBOL Visual aims to organize and systematize such conventions in order to produce a coherent language for expressing the structure and function of genetic designs. At the same time, we aim to make this language simple and easy to use, allowing a high degree of flexibility and freedom in how such diagrams are organized, presented, and styled-in particular, it should be readily possible to create diagrams both by hand and with a wide variety of software programs. Finally, means are provided for extending the language with new and custom diagram elements, and for adoption of useful new elements into the language.

\subsection{Relation to Data Models}

In order to ground SBOL Visual with precise definitions, we reference its visual elements to data models with well-defined semantics. In particular, glyphs in SBOL Visual are defined in terms of their relation to the SBOL 2 data model (as defined in BBF RFC 112) and terms in the Sequence Ontology (Eilbeck et al., 2005), the Systems Biology Ontology (Courtot et al., 2011), and BioPAX (Goldberg et al., 2010).

SBOL Visual is not intended to represent designs at the same level of detail as these data models. Effective visual diagrams are necessarily more abstract, focusing only on those aspects of a system that are the subject of the communication. Nevertheless, we take as a principle that it should be possible to transform any SBOL Visual diagram into an equivalent (if highly abstract) SBOL 2 data representation. Likewise, we require that SBOL Visual should be able to represent all of the significant structural or functional relationships in any GenBank or SBOL data representation.

\section{Relation to other BBF RFCs and other Standards}

SBOL Visual 2.1 replaces BBF RFC 115 (SBOL Visual 2.0).

SBOL Visual 2.1 also implicitly supersedes the previously replaced BBF RFC 93 and BBF RFC 16 (prior versions of SBOL Visual).

Every glyph in SBOL Visual 2.1 corresponds to an element of the SBOL 2.1 data model, as defined in BBF RFC 112. SBOL Visual 2.1 also defines many terms by reference to BBF RFC 112, or by reference to the Sequence Ontology (Eilbeck et al., 2005), the Systems Biology Ontology (Courtot et al., 2011), or BioPAX (Goldberg et al., 2010).

SBOL Visual is intended to be compatible with the Systems Biology Graphical Notation Activity Flow Language (SBGN AF) (Le Novère et al., 2009), and species and interaction glyphs have been imported from that language (see: Appendix A.2 and Appendix A.3). Some aspects are also imported from the Systems Biology Graphical Notation Process Description Language (SBGN PD).

\section{Copyright and License Statement}

Copyright (C) The BioBricks Foundation and all authors listed on this BBF RFC. This work is made available under the Creative Commons Attribution 4.0 International Public License. To view a copy of this license visit https://creativecommons.org/licenses/by/4.0/. 


\section{SBOL Specification Vocabulary}

\subsection{Term Conventions}

This document indicates requirement levels using the controlled vocabulary specified in IETF RFC 2119 and reiterated in BBF RFC 0. In particular, the key words "MUST", "MUST NOT", "REQUIRED", "SHALL", "SHALL NOT", "SHOULD", "SHOULD NOT", "RECOMMENDED", "MAY", and "OPTIONAL" in this document are to be interpreted as described in RFC 2119:

• The words "MUST", "REQUIRED", or "SHALL" mean that the item is an absolute requirement of the specification.

- The phrases "MUST NOT" or "SHALL NOT" mean that the item is an absolute prohibition of the specification.

- The word "SHOULD" or the adjective "RECOMMENDED" mean that there might exist valid reasons in particular circumstances to ignore a particular item, but the full implications need to be understood and carefully weighed before choosing a different course.

- The phrases "SHOULD NOT" or "NOT RECOMMENDED" mean that there might exist valid reasons in particular circumstances when the particular behavior is acceptable or even useful, but the full implications need to be understood and the case carefully weighed before implementing any behavior described with this label.

[ The word "MAY" or the adjective "OPTIONAL" mean that an item is truly optional.

\subsection{SBOL Class Names}

The definition of SBOL Visual references several SBOL classes, which are defined as listed here. For full definitions and explanations, see BBF RFC 112, describing the SBOL 2.1 data model.

ComponentDefinition: Describes the structure of designed entities, such as DNA, RNA, and proteins, as well as other entities they interact with, such as small molecules or environmental properties.

- Component: Pointer class. Incorporates a child ComponentDefinition by reference into exactly one parent ComponentDefinition. Represents a specific occurrence or instance of an entity within the design of a more complex entity. Because the same definition might appear in multiple designs or multiple times in a single design, a single ComponentDefinition can have zero or more parent ComponentDefinitions, and each such parent-child link requires its own, distinct Component.

- Location: Specifies the base coordinates and orientation of a genetic feature on a DNA or RNA molecule or a residue or site on another sequential macromolecule such as a protein.

- SequenceAnnotation: Describes the Location of a notable sub-sequence found within the Sequence of a ComponentDefinition. Can also link to and effectively position a child Component.

- SequenceConstraint: Describes the relative spatial position and orientation of two Component objects that are contained within the same ComponentDefinition.

ModuleDefinition: Describes a "system" design as a collection of biological components and their functional relationships.

- FunctionalComponent: Pointer class. Incorporates a child ComponentDefinition by reference into exactly one parent ModuleDefinition. Represents a specific occurrence or instance of an entity within the design of a system. Because the same definition might appear in multiple designs or multiple times in a single design, a single ComponentDefinition can have zero or more parent ModuleDefinitions, and each such parent-child link requires its own, distinct FunctionalComponent. 
- Interaction: Describes a functional relationship between biological entities, such as regulatory activation or repression, or a biological process such as transcription or translation.

- MapsTo: When a design (ComponentDefinition or ModuleDefinition) includes another design as a sub-design, the parent design might need to refer to a ComponentInstance (either a Component or FunctionalComponent) in the sub-design. In this case, a MapsTo needs to be added to the instance for the sub-design, and this MapsTo needs to link between the ComponentInstance in the sub-design and a ComponentInstance in the parent design.

- Module: Pointer class. Incorporates a child ModuleDefinition by reference into exactly one parent ModuleDefinition. Represents a specific occurrence or instance of a subsystem within the design of a larger system. Because the same definition in multiple designs or multiple times in a single design, a single ModuleDefinition can have zero or more parent ModuleDefinitions, and each such parentchild link requires its own, distinct Module.

- Participation: Describes the role that a FunctionalComponent plays in an Interaction. For example, a transcription factor might participate in an Interaction as a repressor or as an activator. 


\section{SBOL Glyphs}

A glyph is a visual symbol used to represent an element in an SBOL Visual diagram. All of the currently defined glyphs are collected in Appendix A. This section explains how glyphs are specified and how to add new glyphs.

Each SBOL glyph is defined by association with ontology terms, and can be used to represent any diagram element that is well-described by that term. Currently there are three classes of glyphs, each associated with a different ontology and different class in the SBOL 2 data model:

- Sequence Feature Glyphs describe features of nucleic acid sequences. They are associated with Sequence Ontology terms. For the SBOL 2 data model, this is formally defined as any Component with a compatible term within its associated roles, i.e., one that is equal to or a child of at least one term associated with the glyph.

- Molecular Species Glyphs represent any class of molecule whose detailed structure is not being shown using sequence feature glyphs. They are associated with BioPAX terms. For the SBOL 2 data model, this is formally defined as any FunctionalComponent with a compatible term within its associated types, i.e., one that is equal to or a child of at least one term associated with the glyph.

- Interaction Glyphs are "arrows" indicating functional relationships between sequence features and/or molecular species. They are associated with Systems Biology Ontology terms. For the SBOL 2 data model, this is formally defined as any Interaction with a compatible term within its types, i.e., one that is equal to or a child of at least one term associated with the glyph, and with a compatible Participation at the head and tail of the arrow.

More than one glyph may share the same definition: in this case, these glyphs form a family of variants, of which precisely one MUST be designated as the RECOMMENDED glyph, which is to be used unless there are strong reasons to prefer an alternative variant.

It will also frequently be the case that a diagram element could be represented by more than one glyph (e.g., a glyph for a specific term and a glyph for a more general term). In such cases, it is RECOMMENDED that the most specific applicable glyph be used. However, if upward branching in the relevant ontology means two applicable glyphs do not have an ordered parent/child relation, then either MAY be used.

For example, a protein coding sequence (CDS) is a sequence feature that may be represented either using the CDS glyph (Sequence Ontology term SO:0000316) or the Unspecified glyph (Sequence Ontology term SO:0000001). Since SO:0000316 is contained by SO:0000001, the preferred glyph is CDS, rather than Unspecified. Likewise, a CDS may be represented by either a pentagonal glyph or an arrow glyph, but the pentagon is the RECOMMENDED variant, and so it is likewise preferred. Figure 1 illustrates this example.

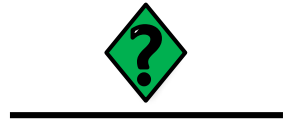

SHOULD NOT

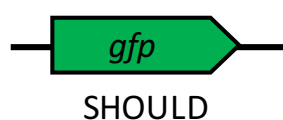

SHOULD

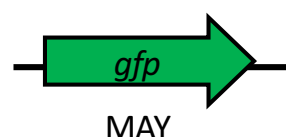

MAY

Figure 1: A biological design element such as a protein coding sequence (CDS) is best represented by the most specific RECOMMENDED glyph (middle), but can be represented by a less specific glyph such as Unspecified (left) or an approved alternative glyph (right). 


\subsection{Requirements for Glyphs}

A number of requirements are placed on all SBOL Visual glyphs in order to ensure both the clarity of diagrams and the ease with which they can be constructed:

1. A glyph SHOULD have its meaning defined by associating the glyph with at least one ontology definition. Definitions are RECOMMENDED to be from the Sequence Ontology for nucleic acid components, from BioPAX for other components, and from the Systems Biology Ontology for interactions. If no applicable terms are available in the preferred ontology, proposal of a new glyph SHOULD be accompanied by a request to the ontology maintainers to add a term for the undefined entity.

2. A glyph SHOULD be relatively easy to sketch by hand (e.g., no high-complexity images or precise angles required).

3. A glyph specification MUST indicate which portions of the glyph are the "interior" for purposes of color fill.

4. A glyph specification SHOULD show the glyph in its preferred relative scale with respect to other glyphs.

5. A glyph SHOULD be specified using only solid black lines (leaving color and style to be determined by the user, as noted below).

6. A glyph SHOULD NOT be similar enough to be easily confused with any other glyph when written by hand, or when scaled either vertically, horizontally, or both.

7. A glyph SHOULD NOT include text (note that associated labels are not part of the glyph).

In addition, some requirements apply only to certain classes of glyphs:

8. A sequence feature or molecular species glyph specification MUST include a rectangular bounding box indicating its extent in space.

9. A sequence feature glyph specification MUST include exactly one horizontal rule for its RECOMMENDED vertical alignment with the nucleic acid backbone.

10. A sequence feature glyph SHOULD be asymmetric on the horizontal axis. Vertical asymmetry is also preferred when possible.

11. If a sequence feature glyph can represent components of highly variable size or structural complexity, the glyph SHOULD be able to be scaled horizontally to indicate relative property value.

Figure 2 shows examples of compliant glyph specification.

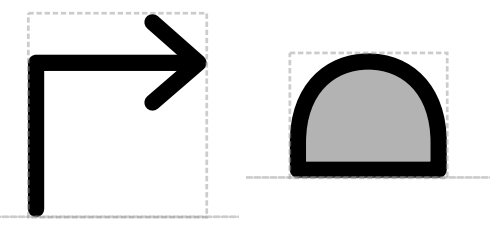

Figure 2: Examples of glyph specification: this specification for the sequence feature glyphs for Promoter (left) and Ribosome Entry Site (right) include the glyph outline, fill (grey center of Ribosome Entry Site), bounding box (dashed box), and recommended alignment with the nucleic acid backbone (dashed horizontal line), all at a preferred relative scale. 


\subsection{Reserved Visual Properties}

SBOL Visual aims to allow as much flexibility and freedom as possible in how diagrams are organized, presented, and styled. To this end, a number of aspects of presentation are generally reserved for the communication of other types of information by the creator of a diagram. When using a glyph in a diagram, the following choices in glyph presentation are thus explicitly intended to be alterable:

1. The lines of a glyph MAY be given any line thickness and style

2. The interior of a glyph MAY be given any fill color, as long as the choice of fill does not interfere with recognizing the glyph.

3. The scale of glyphs are RECOMMENDED to be kept consistent with their specification and throughout a diagram, but can be altered if desired, particularly to convey additional information (e.g., length of a sequence).

4. Minor styling effects MAY be chosen (e.g., shadow, corner styling, other "font-level" customization)

Figure 3 shows some examples of acceptable style variation.

In certain special cases, the style of a glyph may be more constrained, but such cases are expected to be rare and strongly motivated.

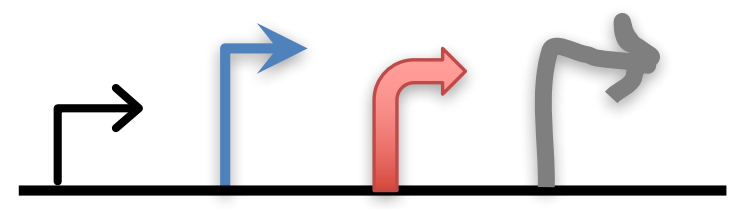

Figure 3: Examples of acceptable style variation for a Promoter glyph.

\subsection{Extending the Set of Glyphs}

The collection of SBOL Visual glyphs is not expected to provide complete coverage of all of the types of element that people will wish to include in genetic diagrams, particularly given the ongoing evolution of synthetic biology as an engineering discipline. As the need for new diagram elements or new practices of usage emerge, new glyphs or glyph definitions are expected to be added to SBOL Visual. In particular, the following three classes of changes are expected to occur regularly, and the SBOL development community will maintain clear processes for proposal and adoption of changes of this type:

- New glyphs, either representing a type of component that previously lacked a glyph or enabling a distinction between types of components previously represented by the same glyph.

- Additional glyph variants, accompanied by compelling use cases that cannot be adequately addressed by the existing glyph variants.

- Additional definitions for a glyph, capturing an alternate meaning that is useful to humans but existing within a disjoint branch of the relevant ontology.

In order to support the coherent extension of SBOL Visual, whenever a diagram creator uses a glyph not found in Appendix A, the creator SHOULD submit it to be considered for inclusion in an updated version of the standard following the processes for adding new glyphs found on the community website at http: //sbolstandard. org 


\section{SBOL Visual Diagram Language}

An SBOL Visual diagram represents information about the structure of a nucleic acid design and its associated molecular species and interactions. If desired, an SBOL Visual diagram may also be associated with a machineinterpretable model (e.g., in SBOL, GenBank, or SBML format). In this document we describe the association for the SBOL 2 data model, which provides a formal semantic grounding for all elements of an SBOL Visual diagram, but equivalent associations may be made between diagram elements and other models. In terms of the SBOL 2 data model, the description of a nucleic acid design is formally defined as a representation of a ComponentDefinition with a nucleic acid type, the Component and SequenceAnnotation objects describing the features and sub-structure of the design, and SequenceConstraint information on the relative positions of such elements. The description of interactions between some number of nucleic acid designs and other molecular species is formally defined as a representation of a ModuleDefinition, the FunctionalComponent objects describing the nucleic acid designs and other molecular species, and the Interaction and Participation objects describing their functional relationships.

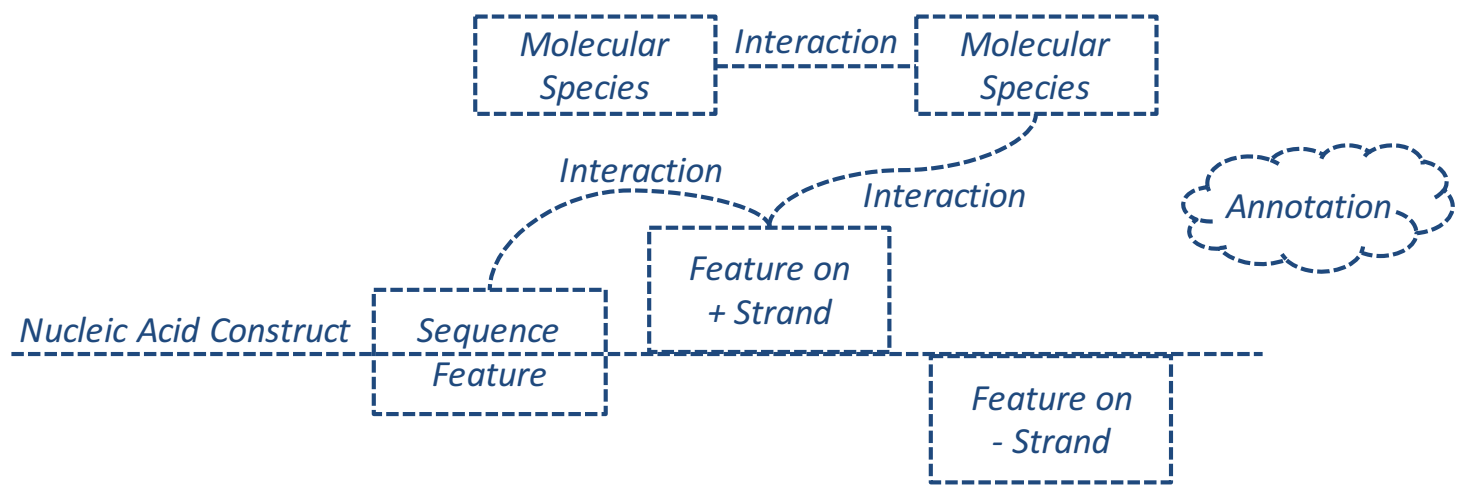

Figure 4: Generic syntax of SBOL Visual 2: a diagram for a nucleic acid construct is based around a backbone line, its structure specified by the sequence of attached sequence feature glyphs. Strand can optionally be indicated by placing a glyph above or below the backbone. Other molecular species are indicated by glyphs not in contact with any backbone. Interactions are directed edges connecting sequence feature or molecular species glyphs. Any of these objects may have an associated label showing its name, and the diagram may further include any form of other annotations, including other types of text.

Specifically, an SBOL Visual diagram consists of the classes of objects illustrated in Figure 4. Figure 5 shows an example of such a diagram, in a typical usage. Full details of this specification are provided in the remainder of this section.

\subsection{Nucleic Acid Backbone}

A diagram for a nucleic acid construct is based around a single or double line, representing the nucleic acid backbone. Information about features of the construct can then be represented by attaching nucleic acid glyphs to the backbone, as defined below in Section 6.2. In terms of the SBOL 2 data model, the backbone represents a ComponentDefinition with a nucleic acid type (e.g., DNA, RNA), and the features represent Component and SequenceAnnotation members of the ComponentDefinition.

1. Lines in some cases indicate strand count. A double-stranded region of the nucleic acid construct MAY use either a single or double line for the backbone. A single-stranded region of the nucleic acid construct MUST use a single line to indicate the backbone. When single and double lines are mixed within a single diagram, the single lines always indicate single-stranded regions. Examples are provided in Figure 6. 


\section{Sequence Feature Glyphs}

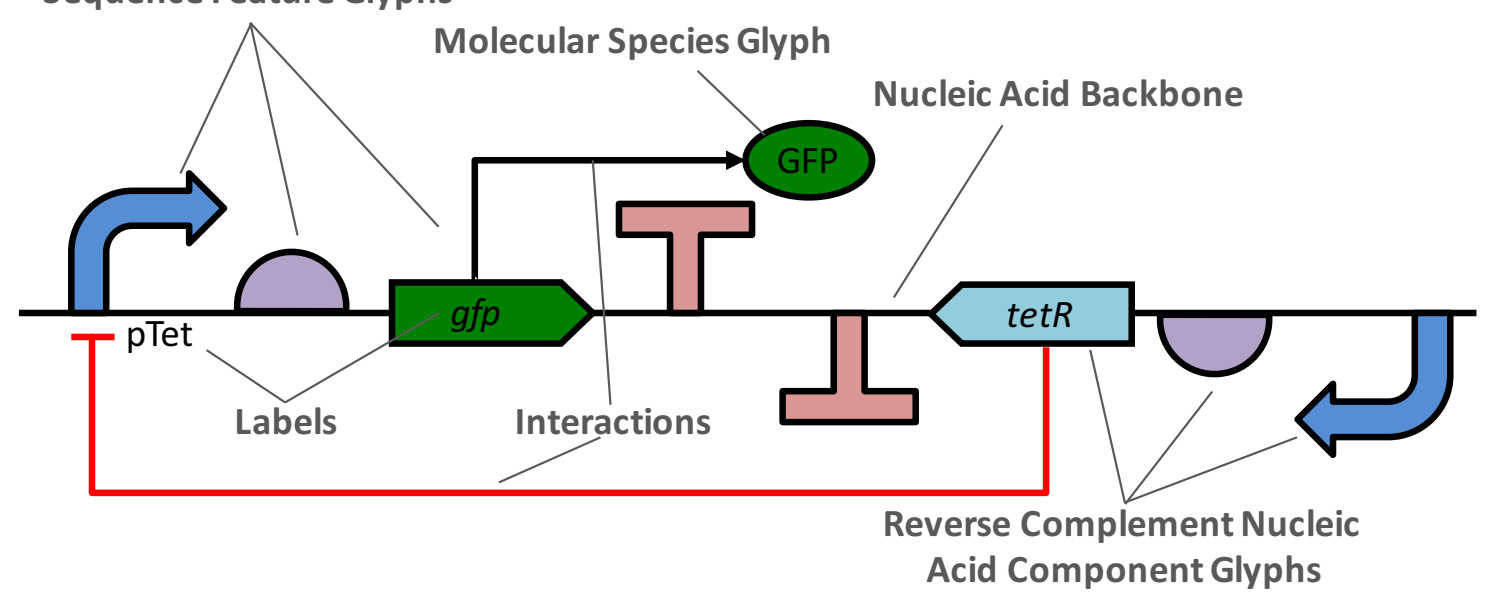

Figure 5: Example illustrating the elements of an SBOL Visual 2 diagram, with nucleic acid sequence features on the forward and reverse strand of a backbone, other molecular species, and interactions between elements; the grey labels and indicator lines are annotations.

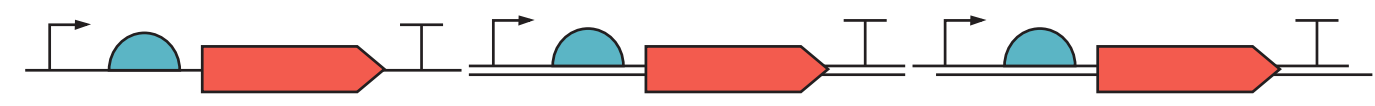

(a) Single- or double-strand backbone

(b) Double-strand backbone

(c) Double-strand backbone with singlestrand overhangs

Figure 6: Examples of indicating strand count in nucleic acid backbones.

2. A nucleic acid backbone SHOULD be horizontal in orientation, but MAY use non-horizontal structure to indicate important physical attributes (e.g., a closed loop to indicate a cyclic plasmid or more complex shapes for DNA nanotech structures). Examples are provided in Figure 7.

3. As a special case of non-horizontal backbone structure, certain stylized backbone shapes are used as sequence feature glyphs to indicate the genomic context of a sequence. These glyphs SHOULD be used as a matched pair, indicating the bounds of the context region. It is further RECOMMENDED that each glyph be concatenated with an Omitted Detail glyph to explicitly indicate that some surrounding context is not being shown. Examples are provided in Figure 8.

4. A nucleic acid backbone SHOULD have at least one associated feature glyph (else no structural information is being provided).

\subsection{Nucleic Acid Sequence Features}

A glyph in contact with a nucleic acid backbone indicates a feature of the nucleic acid sequence. In terms of the SBOL 2 data model, this is either a SequenceFeature or a Component with a nucleic acid type that is contained within the ComponentDefinition associated with that nucleic acid backbone. The Component may be contained either directly, as one of the components of the ComponentDefinition, or recursively through a sequence of such containments.

1. Every feature glyph MUST have its bounding box in contact with the backbone for the nucleic acid construct it describes. The placement of the glyph SHOULD follow the recommendation for backbone alignment in the glyph specification. Examples are provided in Figure 9.

2. The horizontal orientation of a glyph can be used to indicate the strand alignment of a feature, as shown in 


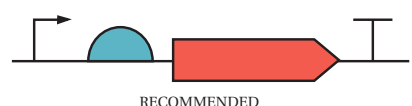

RECOMMENDED
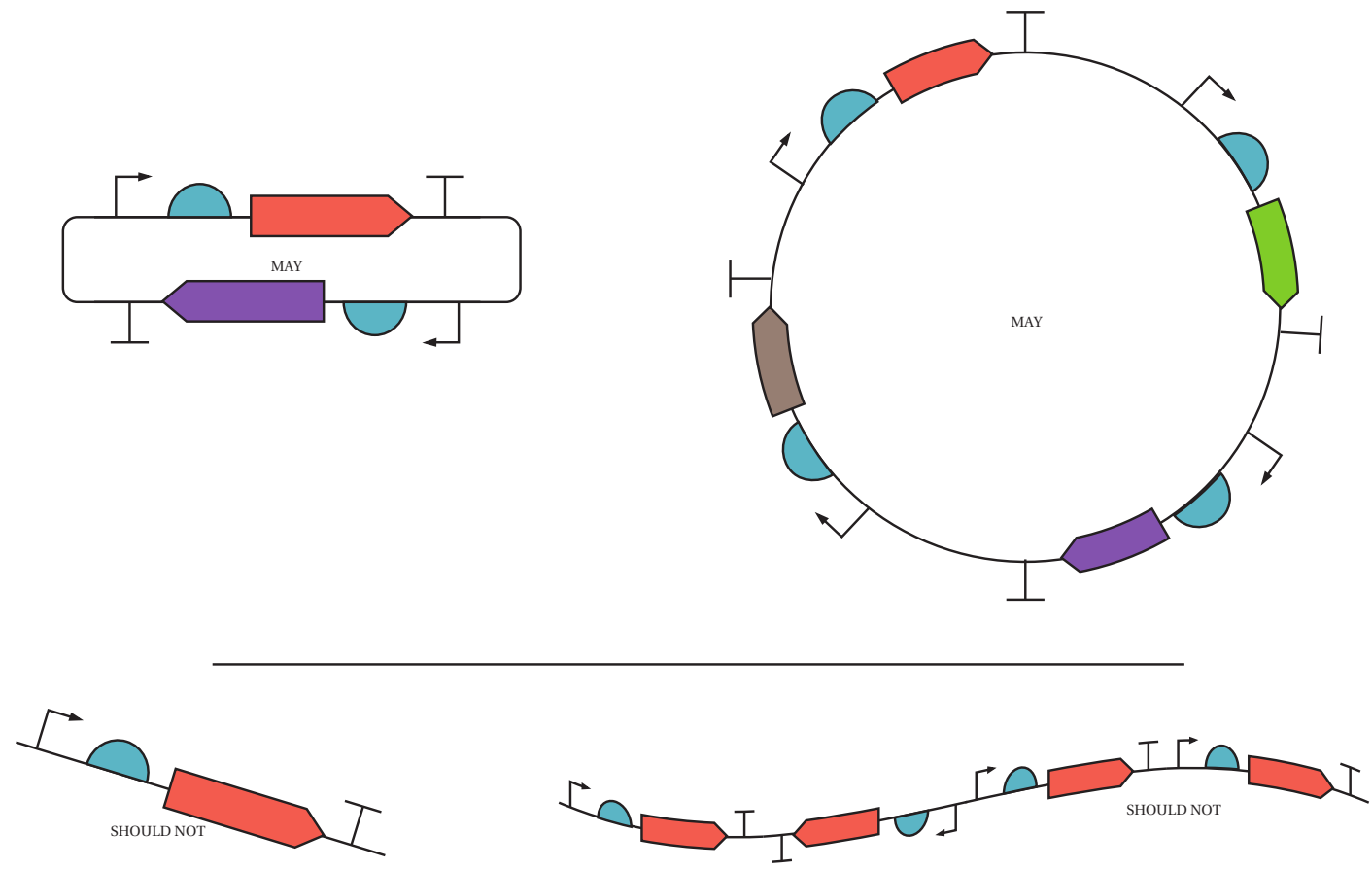

Figure 7: Recommended, acceptable, and problematic examples of nucleic backbone orientation.

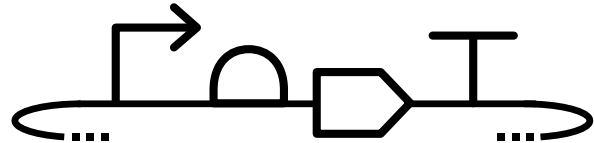

(a) Functional unit on a circular plasmid

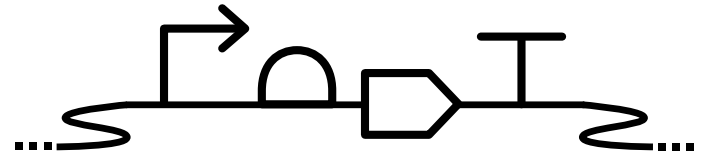

(b) Functional unit integrated at a chromosomal locus

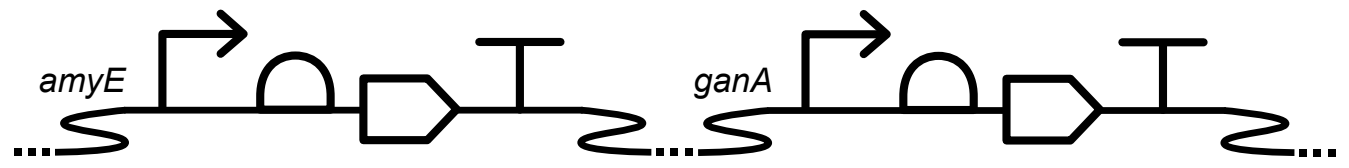

(c) Two functional units on the same chromosome but different loci

Figure 8: Examples of RECOMMENDED indication of genomic context.

Figure 10. Any glyphs for a feature associated with the inline strand SHOULD be placed in the prototypical orientation given by the specification, while any glyph that is associated with the reverse complement strand SHOULD be inverted vertically and horizontally (i.e., rotated 180 degrees). Reverse complement MAY also be 


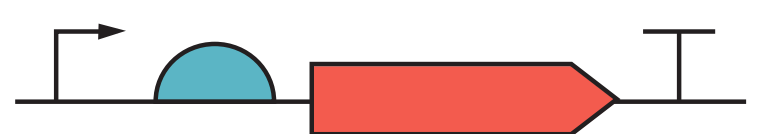

(a) MUST

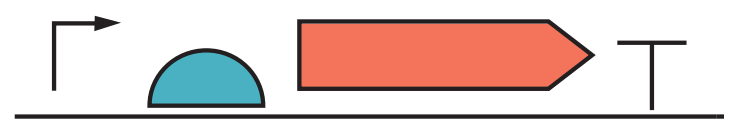

(b) MUST NOT

Figure 9: Examples of correct and incorrect association of glyphs with a nucleic acid backbone.

indicated by horizontal-only inversion. Finally, a glyph inverted only vertically still indicates inline strand, but it is RECOMMENDED NOT to use this orientation. Orientation SHOULD be used consistently throughout a diagram, rather than mixing conventions. Examples are provided in Figure 11.

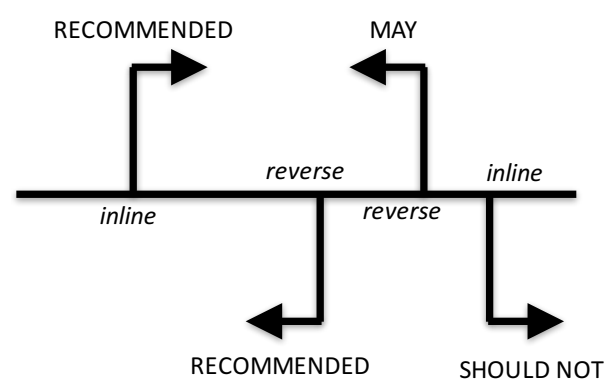

Figure 10: Use of glyph orientation to indicate inline vs. reverse complement direction.

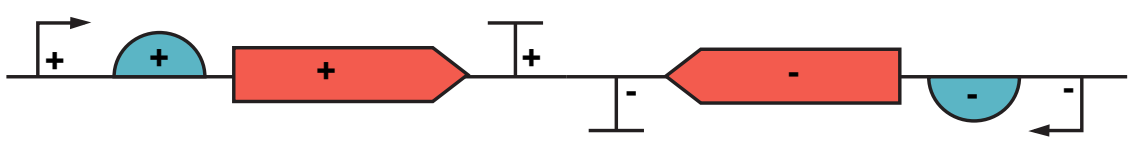

Figure 11: Example construct incorporating both inline (+) and reverse complement (-) features.

3. Nucleic acid features in a sequential relationship SHOULD be drawn from 5' left to 3' right on the inline strand and from 5' right to 3' left on the reverse complement strand. In terms of the SBOL 2 data model, this indicates a SequenceConstraint on the relative ordering of two features.

4. Nucleic acid features that do not overlap in their locations SHOULD NOT have glyphs whose bounding boxes overlap. An example is provided in Figure 12.

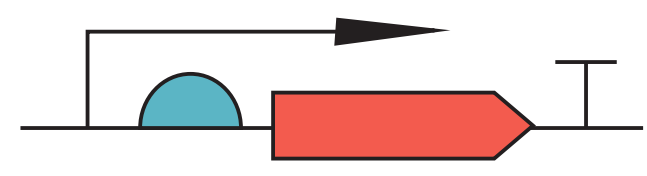

Figure 12: Example of incorrect glyph overlap: promoter (arrow) does not overlap in sequence with the ribosome entry site and CDS, so SHOULD NOT overlap visually with them. 
5. Nucleic acid features that overlap in their locations SHOULD have glyphs whose bounding boxes overlap. Overlap size MAY be used to indicate relative position. Examples are provided in Figure 13.

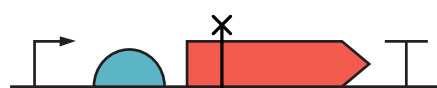

(a) Restriction site in a CDS

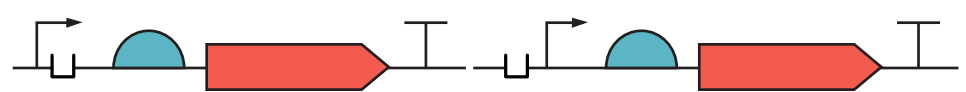

(b) 3'-side operator in a promoter (c) 5'-side operator in a promoter

Figure 13: Examples where glyphs SHOULD overlap, but might not if it is more clear, e.g., with an operator site located within the 5' portion of a promoter.

6. A nucleic acid feature SHOULD be represented using a glyph defined in Appendix A.1. In this case, the feature MUST be contained within at least one of the glyph's associated terms. In terms of the SBOL 2 data model, this means the glyph is equal to or a parent of at least one of the roles for the Component or its associated ComponentDefinition. Moreover, the glyph used SHOULD be the RECOMMENDED variant of the most specific applicable glyph. Note that novel glyphs not defined in Appendix A.1 MAY be used, but SHOULD be proposed for adoption as described in Section 5.3. Examples are provided in Figure 14.

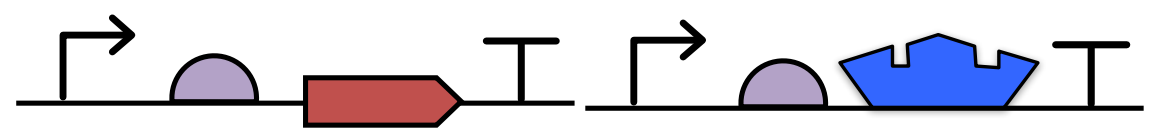

(a) SHOULD

(b) MAY

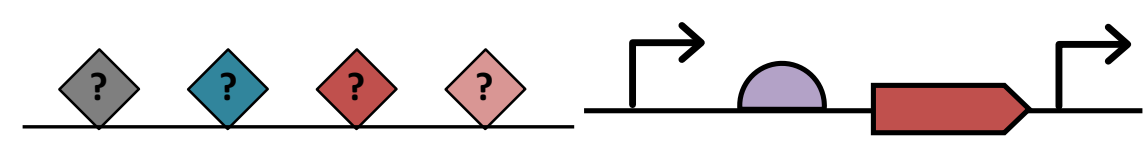

(c) SHOULD NOT

(d) MUST NOT

Figure 14: Examples of recommended, allowed, and forbidden representation of a ComponentDefinition comprising a sequence of promoter, ribosome entry site, CDS, and terminator: (a) is RECOMMENDED because it uses the preferred variant of the most specific defined glyphs, (b) is allowed because it uses some novel custom non-conflicting symbol, not matching any glyph defined in this document, to encode more specific information about the particular CDS, (c) is recommended against because it uses less specific glyphs, and (d) is forbidden because it use a promoter symbol to represent the terminator.

\subsection{Molecular Species}

A glyph that is not in contact with any backbone represents any class of molecule whose detailed structure is not being shown using sequence feature glyphs. In other words, either not a nucleic acid (e.g., proteins, small molecules) or else an "uninteresting" nucleic acid (e.g., showing a transcribed mRNA, but not the features of its sequence). In terms of the SBOL 2 data model, this is a FunctionalComponent that is contained within a ModuleDefinition implicit in the diagram.

1. A molecular species glyph MUST NOT contact any nucleic acid backbone with any part of its bounding box.

2. A molecular species SHOULD be represented using a glyph defined in Appendix A.2. In this case, the species MUST be contained within at least one of the glyph's associated terms. In terms of the SBOL 2 data model, this means the glyph is equal to or a parent of at least one of the types for the associated ComponentDefinition Moreover, the glyph used SHOULD be the RECOMMENDED variant of the most specific applicable glyph. 
Note that novel glyphs not defined in Appendix A.2 MAY be used, but SHOULD be proposed for adoption as described in Section 5.3.

\subsection{Interaction}

A directed edge "arrow" attached to one or more glyphs indicates a functional interaction involving those elements. The roles of the elements is indicated by their position at the head or tail of the edge. In terms of the SBOL 2 data model, this is an Interaction, with either one or two Participation relationships, their role set by position at the head or tail of the edge. An example is provided in Figure 15.

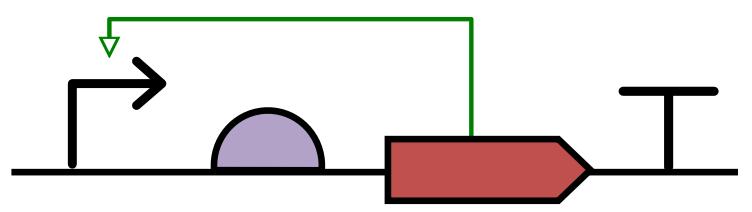

Figure 15: Example of an interaction indicating a promoter stimulated by the CDS that it regulates.

1. Two interaction edges SHOULD NOT cross one another. When edges cross, they MUST indicate the distinction between arrows with a crossover pattern, in which one edge "diverts" at the intersection (see Figure 16). Examples are provided in Figure 17.
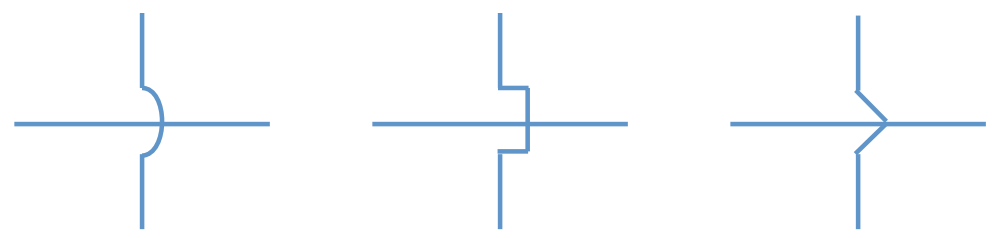

Figure 16: Examples of Interaction crossover patterns.

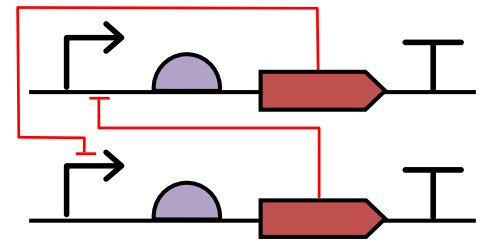

(a) SHOULD

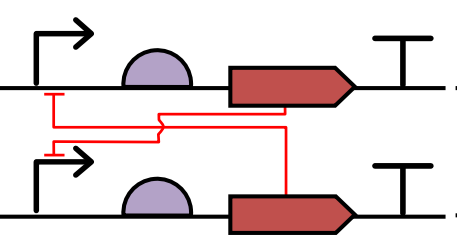

(b) MAY

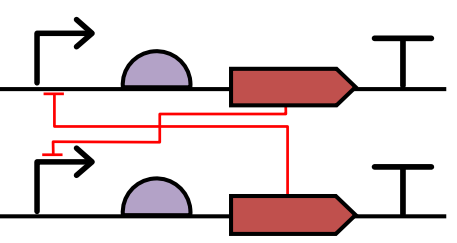

(c) MUST NOT

Figure 17: Examples of recommended, allowed, and forbidden relationships between two interactions in a mutual repression system: (a) non-crossing is recommended, (b) using a crossover pattern is allowed, but (c) crossing without a crossover pattern is forbidden, since the relationship between the two edges is ambiguous.

2. An interaction SHOULD be represented using a glyph defined in Appendix A.3. In this case, the interaction type MUST be contained within at least one of the glyph's associated terms. In terms of the SBOL 2 data model, this means the glyph is equal to or a parent of at least one of the types for the Interaction, and that each associated Participation object has a role compatible with its position on the head or tail of the edge. Moreover, the glyph used SHOULD be the RECOMMENDED variant of the most specific applicable glyph. Note that novel glyphs not defined in Appendix A.3 MAY be used, but SHOULD be proposed for adoption as described in Section 5.3. 
3. An edge may have multiple heads or multiple tails. In this case, a split or join in an edge represents either multiple participants with the same role (e.g., a transcription factor repressing two instances of a promoter) or a biochemical process (e.g., association of an inducible protein and a small molecule to form an active complex). An edge with multiple heads MUST use the same glyph for each head. An edge that splits or joins with no glyph at the junction represents multiple participants with the same role. A glyph at the point where an edge splits or joins represents a biochemical process, i.e., an additional Interaction with type and roles set by the process glyph. Examples are provided in Figure 18.

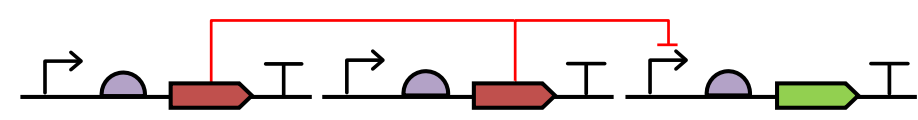

(a) MAY

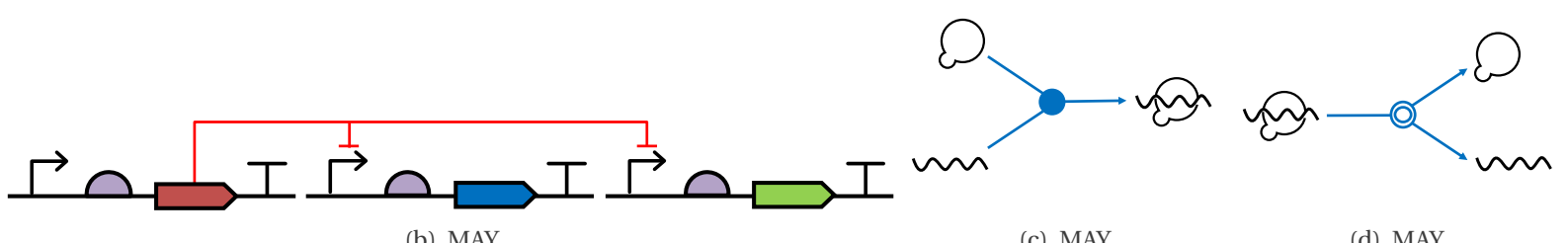

(b) MAY

(c) MAY

(d) MAY

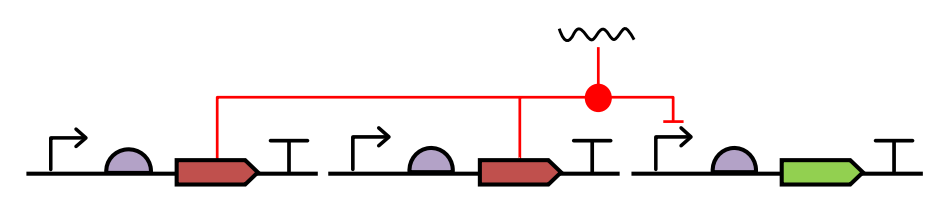

(e) MAY

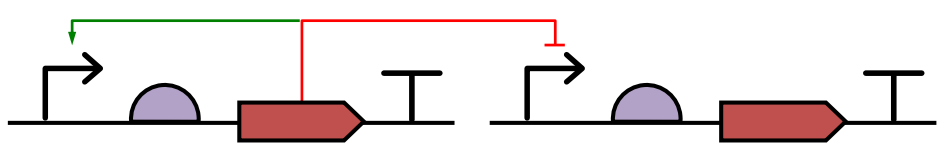

(f) MUST NOT

Figure 18: Examples of use of multi-head and multi-tail arrows: (a) Repression from multiple independent sources, (b) repressor with multiple targets, (c) association of gRNA and Cas9 into an active CRISPR complex and (d) the dissociation of that complex, and (e) composite edges representing two interactions: CRISPR complex formation with Cas9 from two sources, which then represses a promoter. (f) Multi-head interactions, however, MUST NOT use different glyphs for different heads.

4. A biochemical process represented by a glyph at an edge junction SHOULD be represented using a glyph defined in Appendix A.4. In this case, the interaction type MUST be contained within at least one of the glyph's associated terms. In terms of the SBOL 2 data model, this means the glyph is equal to or a parent of at least one of the types for the Interaction, and that each associated Participation object has a role compatible with its position on the head or tail of the edge. Moreover, the glyph used SHOULD be the RECOMMENDED variant of the most specific applicable glyph. Note that novel glyphs not defined in Appendix A.4 MAY be used, but SHOULD be proposed for adoption as described in Section 5.3.

\subsection{Modules}

A module within a system MAY be represented by a visual boundary in the form of closed polygon or closed curve. Everything inside of the boundary is part of the module, and everything outside of the boundary is not part of the module; only certain diagram elements are allowed to cross a boundary, as defined below. In terms of the SBOL 2 data model, the line represents a Module included within the ModuleDefinition represented by the surrounding diagram, and boundary-crossing elements define MapsTo relationships. Note that the internals of a module need 
not be shown: some details can be omitted or a module can even be a "black box" with no internal structure at all being shown.

1. The boundary of module SHOULD should be a rectangle or rounded rectangle. Boundary sides SHOULD be oriented vertically and horizontally. It is RECOMMENDED that a module be made visually distinct by making it larger than other glyphs and with a different line style. Examples are provided in Figure 19.

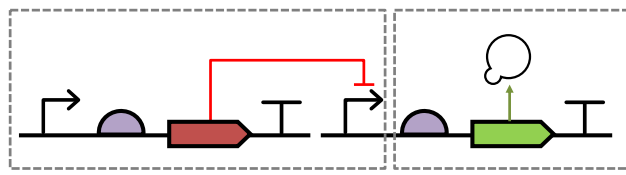

(a) SHOULD

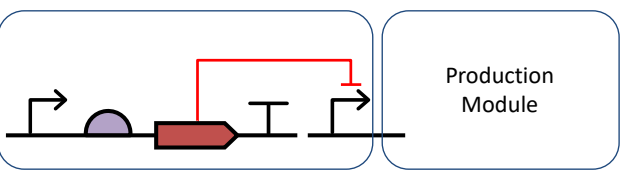

(b) SHOULD

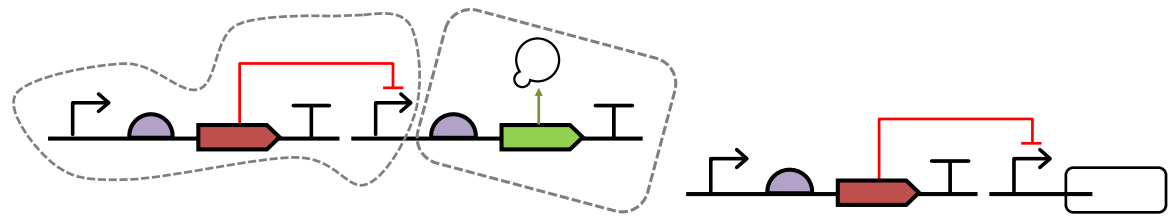

(c) SHOULD NOT

(d) SHOULD NOT

Figure 19: Examples of recommended and problematic module boundaries: (a) two modules with visually distinct rectangular borders, (b) shows the same modules but with rounded rectangles and the second being a "black box" module with no internal structure shown, (c) shows modules with non-rectilinear borders, and (d) shows a black-box module that is not visually distinct from a sequence feature glyph.

2. An undirected edge (i.e., having no "arrow head") that crosses the boundary of a module represents a mapping associating the diagram elements that it links. Glyphs associated by a mapping MUST either be sequence features, molecular species, or module ports (see below), and must be of compatible types. In terms of the SBOL 2 data model, the line represents a MapsTo relationship between a FunctionalComponent in the ModuleDefinition and another FunctionalComponent in the definition of the Module. Mapping edges SHOULD be made visually distinct from other lines in the same diagram, and it is RECOMMENDED that this distinction be made using dashed lines to represent mapping edges. Examples are provided in Figure 20.

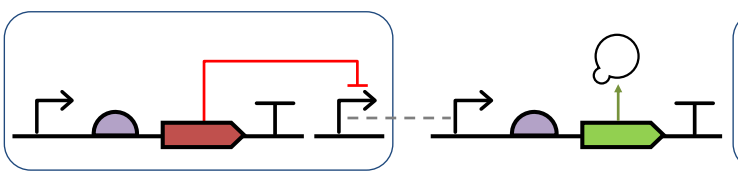

(a) MAY

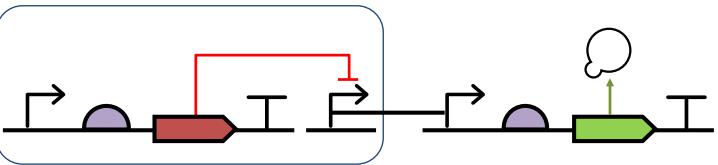

(b) SHOULD NOT

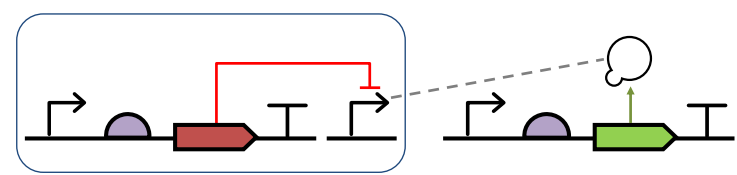

(c) MUST NOT

Figure 20: Examples of recommended and problematic mappings: (a) mapping showing that the promoter inside the module on the left is also used in the construct on the right, (b) mapping is not visually distinct from nucleic acid backbone, (c) mapping cannot identify a promoter with a macromolecule species.

3. Glyphs for sequence features and molecular species MUST NOT intersect with the boundary of a module. A 
nucleic acid backbone MAY cross the boundary of a module. This represents an implicit mapping between the region of the nucleic acid construct contained within the module and a compatible region of the larger construct represented in the enclosing system. An interaction edge MAY cross the boundary of a module. This represents an interaction in the enclosing system plus an implicit mapping between the component inside of the module and a compatible instance in the enclosing system. Examples are provided in Figure 21.

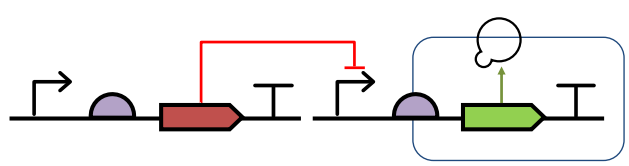

(a) MUST NOT

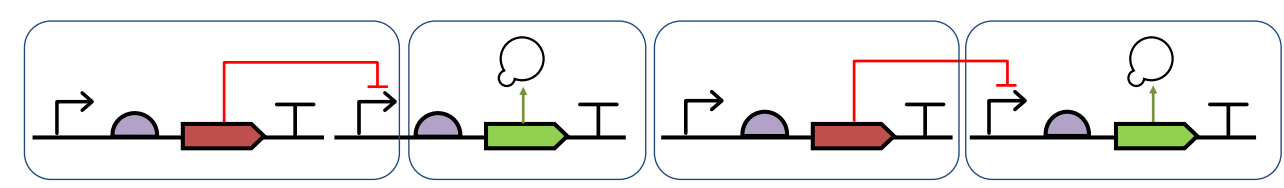

(b) MAY

(c) MAY

Figure 21: Examples of recommended and problematic boundary intersections: (a) sequence feature and molecular species glyphs MUST NOT intersect a module boundary, (b) implicit mapping from the promoter in the left module and the regulated elements in the right module to a nucleic acid construct ordering both into a complete functional unit, (c) implicit mapping from the CDS in the left module and the promoter in the right module to instances in the complete system in which the CDS inhibits the promoter (presumably by a repressor product).

4. Small rectangles MAY be drawn on the outside of the module boundary to represent input/output ports. In terms of the SBOL 2 data model, each rectangle is associated with a FunctionalComponent with a direction property that is in, out, or inout. A port may be connected to an interaction edge head or tail to represent interactions with its associated component. If both a port and a glyph for its associated component are present in a diagram, then they MUST be visually connected, either explicitly by means of a mapping or implicitly by an interaction that passes through the port rectangle. Likewise, mappings and interactions with the associated component MUST NOT cross the boundary except through the port. A port SHOULD NOT both have interactions both connecting to it and crossing the boundary across it. Examples are provided in Figure 22.

\subsection{Labels}

The name of any object in a diagram is RECOMMENDED to be displayed as text within, adjacent to, or otherwise clearly visually connected to the object's associated glyph. In terms of the SBOL 2 data model, this is the name property, and if no name is supplied then the displayId MAY be used instead. Examples are provided in Figure 23.

\subsection{Annotations}

Other text or graphics may be included as annotations with no constraint on their syntax or semantics.

1. Annotations SHOULD NOT be displayed in a way that allows them to be confused with other SBOL Visual elements.

2. Annotations SHOULD NOT be used to display information that can be displayed using other SBOL Visual elements. 


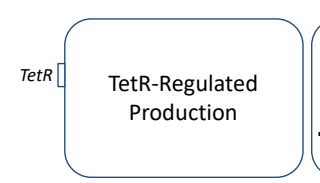

(a) MAY

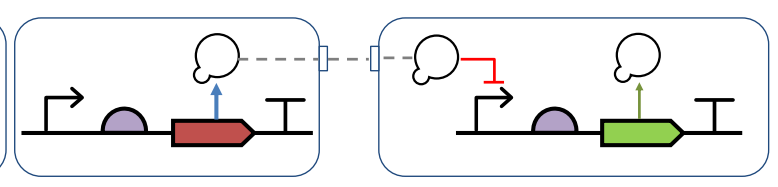

(b) MAY

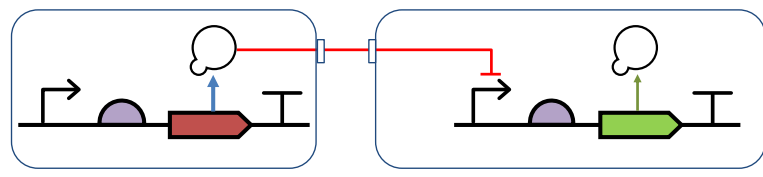

(c) MAY

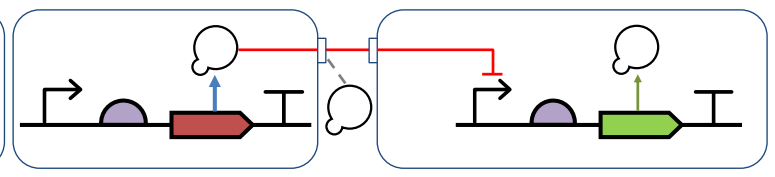

(d) SHOULD NOT

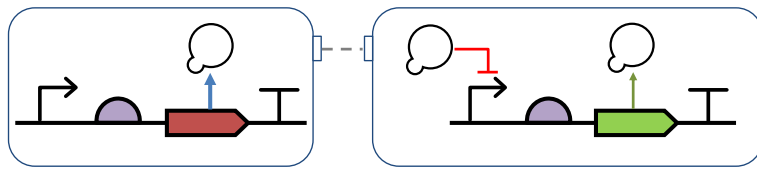

(e) MUST NOT

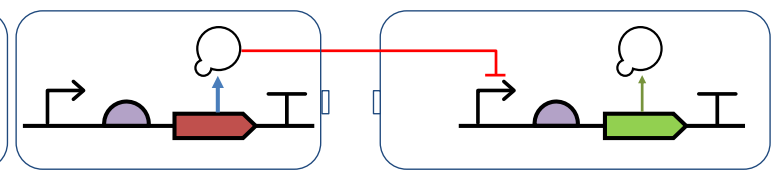

(f) MUST NOT

Figure 22: Examples of recommended and problematic module ports: (a) port on a black-box module, (b) ports connected to module internals via mappings, (c) boundary-crossing interaction passing through a port, (d) diagrams SHOULD NOT mix mappings and interactions on a give port, (e) ports MUST NOT be disconnected from their associated glyphs, and (f) if a port exists, interactions with that port MUST NOT cross the boundary at any other location.

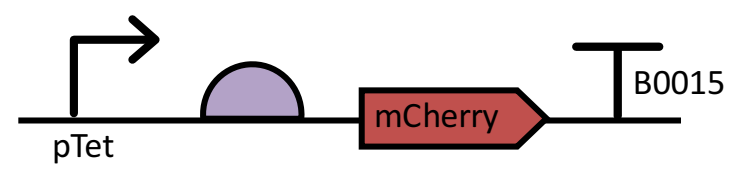

Figure 23: Examples of labels on glyphs.

\subsection{Criteria for Compliance with SBOL Visual}

A diagram of a biological system is compliant with SBOL Visual if it complies with all MUST and MUST NOT requirements as specified above. A diagram is compliant with SBOL Visual best practices if it also complies with all RECOMMENDED, SHOULD, and SHOULD NOT statements as specified above.

Importantly, note that a non-SBOL glyph can be used in a compliant diagram when its definition is a subset or superset of a definition that does have an SBOL Visual glyph. For example, a diagram that creates a new glyph for a special type of promoter can be SBOL Visual compliant even though there is an SBOL Visual glyph for a general promoter.

A piece of software or other system for producing diagrams is compliant with SBOL Visual under the following conditions:

1. The system MUST be capable of producing diagrams that are compliant with SBOL Visual.

2. If the system can also produce diagrams that are not compliant with SBOL Visual, it MUST clearly distinguish to the user between compliant and non-compliant usage and diagrams. 


\section{A SBOL Visual Glyphs}

The following pages present all current glyphs for SBOL Visual, organized by glyph families. Each entry lists:

- Glyph family name

- Associated ontology terms

- Recommended and alternate glyphs

At least one example of when this glyph would be used

- Any additional notes

\section{A.1 Sequence Feature Glyphs}

These glyphs represent features of nucleic acid sequences, and include a bounding box (grey dashed box) and a recommended alignment to the nucleic acid backbone (grey dashed horizontal line). 


\section{Aptamer}

\section{Associated SO term(s)}

SO:0000031: Aptamer

\section{Recommended Glyph and Alternates}

The aptamer glyph is a cartoon diagram of a prototypical nucleic acid secondary structure for an aptamer:

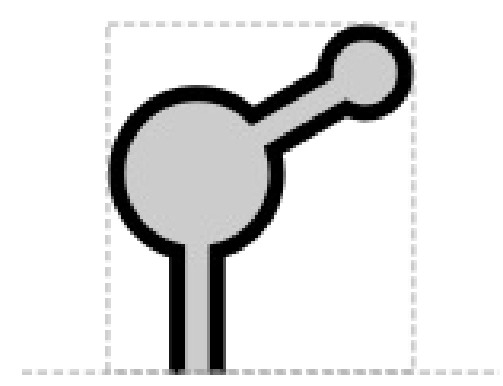

\section{Prototypical Example}

theophylline aptamer

\section{Notes}

this section deliberately blank 


\section{Assembly Scar}

\section{Associated SO term(s)}

SO:0001953

\section{Recommended Glyph and Alternates}

The assembly scar glyph is an "equal sign" image, the pattern produced by the union of a 5 ' sticky end and 3' sticky end glyph. The scar will cover the backbone, creating a visual break suggesting the potential disruption associated with a scar:

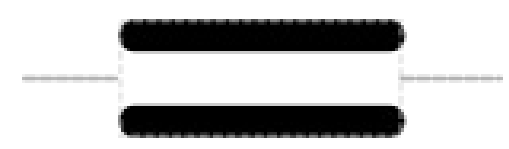

With a double-stranded backbone:

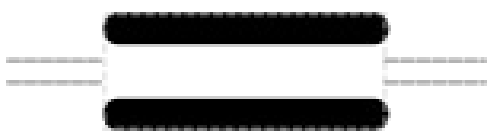

\section{Prototypical Example}

Ligated sticky ends following BioBrick assembly.

\section{Notes}

this section deliberately blank 


\section{Blunt Restriction Site}

\section{Associated SO term(s)}

SO:0001691

\section{Recommended Glyph and Alternates}

The blunt restriction site glyph is an image of two brackets facing away from one another to make a smooth-edged gap:

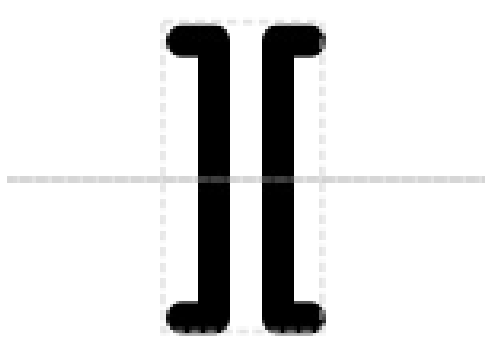

\section{Prototypical Example}

EcoRV restriction site

\section{Notes}

this section deliberately blank 


\section{CDS}

\section{Associated SO term(s)}

SO:0000316

\section{Recommended Glyph and Alternates}

The coding sequence glyph is a "box" with one side bent out arrow-like to show direction:

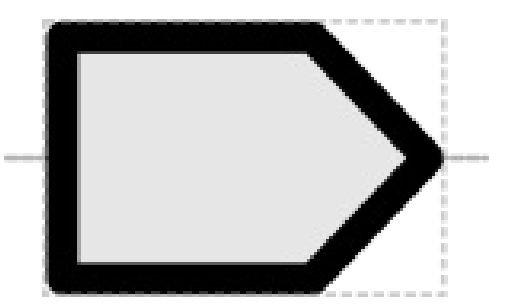

Alternately, CDS may be represented as a block arrow:

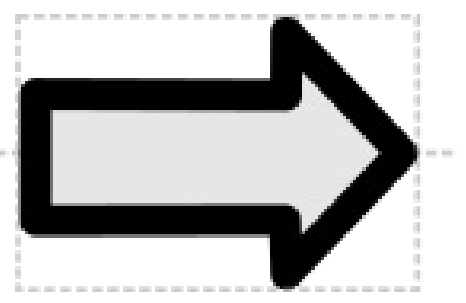

\section{Prototypical Example}

$\alpha$-Hemoglobin coding sequence

\section{Notes}

this section deliberately blank 


\section{Chromosomal Locus}

\section{Associated SO term(s)}

SO:0000830 Chromosome Part

\section{Recommended Glyph and Alternates}

The glyph to indicate integration into a chromosome is an S-shaped curve of the backbone, suggesting something that might be part of a larger looping structure:

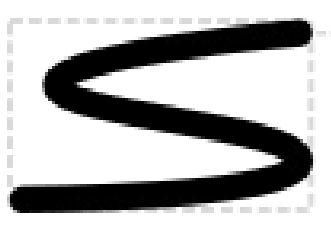

\section{Prototypical Example}

B. subtilis amyE locus

\section{Notes}

Complementary "left" and "right" versions of this glyph SHOULD be used together, flanking the region whose genomic context is being described.

The Omitted Detail glyph SHOULD generally be contatenated to indicate that there is information about the chromosome not being represented.

Examples of RECOMMENDED usage:

- A functional unit consisting of promoter, ribosome entry site, CDS, and terminator, all integrated together into the chromosome:

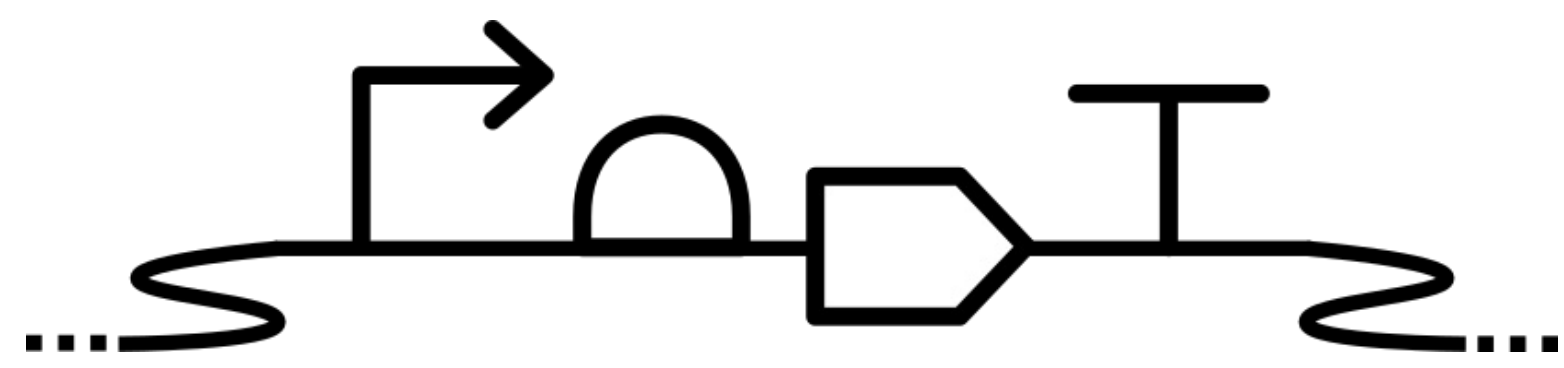

- Two functional units, one integrated into the amyE locus, another integrated into the ganA locus:

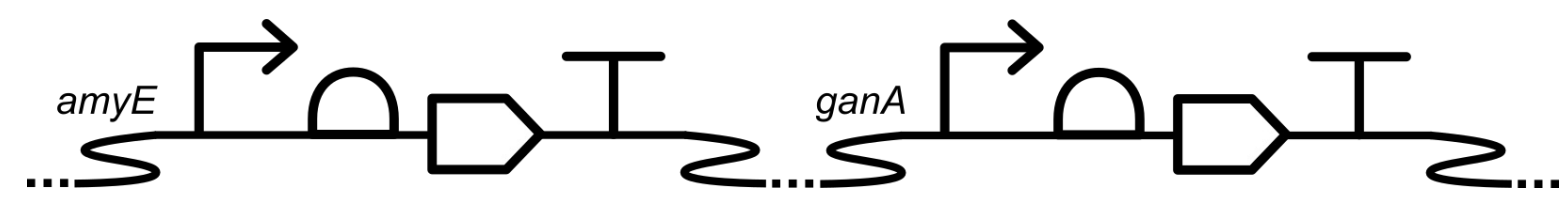




\section{Circular Plasmid}

\section{Associated SO term(s)}

SO:0002211 Circular Plasmid - A self replicating circular nucleic acid molecule that is distinct from a chromosome in the organism.

\section{Recommended Glyph and Alternates}

The glyph to indicate embedding in a plasmid is a turn of the backbone indicating its circular structure:

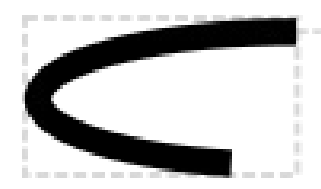

\section{Prototypical Example}

E. coli p15A plasmid

\section{Notes}

Note that for SBOL data representations, circularity SHOULD also be indicated with a type of SO:0000988.

Complementary "left" and "right" versions of this glyph SHOULD be used together, flanking the region whose genomic context is being described.

The Omitted Detail glyph SHOULD generally be concatenated to indicate that there is information about the plasmid not being represented.

Example of RECOMMENDED usage: a plasmid containing a functional unit consisting of promoter, ribosome entry site, CDS, and terminator:

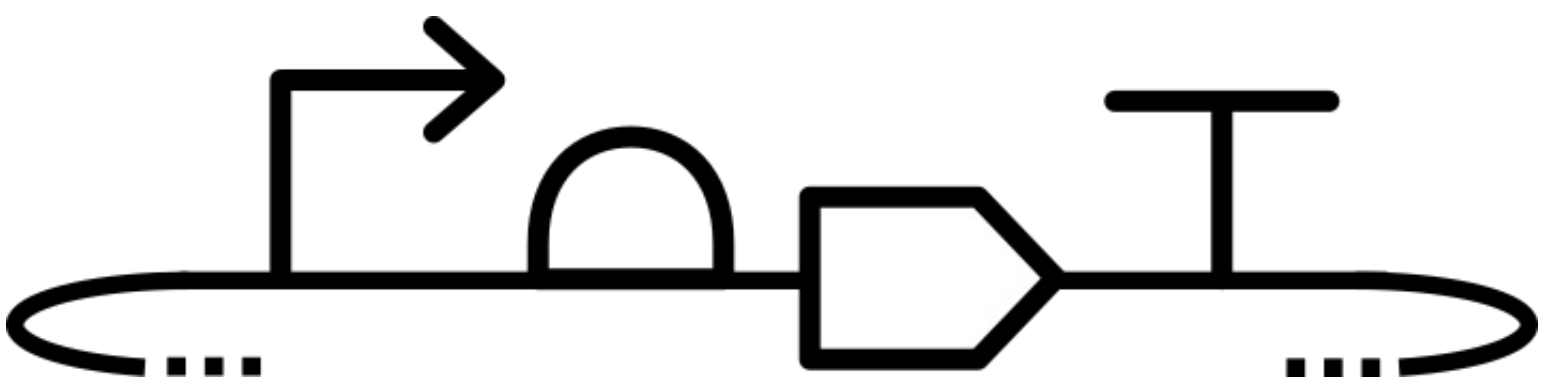




\section{Cleavage Site}

\section{Associated SO term(s)}

SO:0001956 (Protease Site)

SO:0001977 (Ribonuclease Site)

SO:0001688 (Restriction Enzyme Cleavage Junction), SO:0001687 (Restriction Enzyme Recognition Site)

\section{Recommended Glyph and Alternates}

Cleavage Site is a "stem-top" glyph for describing small sites. In this system:

- the top glyph indicates the type of site (e.g., Cleavage Site)

- the stem glyph indicates whether the site affects DNA, RNA, or protein (respectively: straight, wavy, or looped)

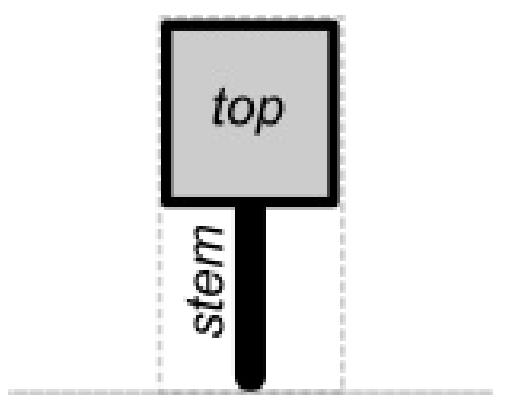

The Cleavage Site top is an "X" suggesting slicing on top of a stem connecting to the backbone at the point where cleavage will occur (in order: DNA, RNA, Protein): 


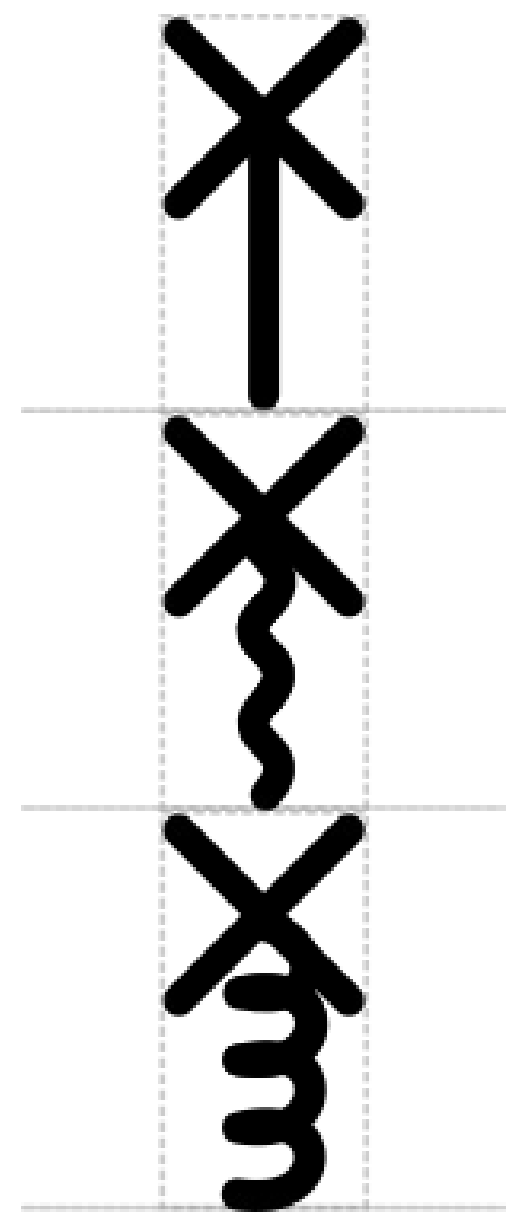

\section{Prototypical Example}

RNAse E site, BamHI

\section{Notes}

SO:0000061 (which was previously associated with Restriction Enzyme Recognition Site in SBOL Visual 1.0) is no longer associated with the DNA Cleavage glyph in SBOL Visual 2, as SO:0000061 refers to the binding site and not the location of cleavage.

The Ribonuclease Site, Protease Site, and Restriction Enzyme Recognition Site glyphs from SBOL Visual 1.0 are now replaced by the Cleavage Site glyph with the appropriate stem.

Describing a Restriction Enzyme Cleavage Site with a vertical line glyph on a DNA backbone (as done previously in SBOL Visual 1.0 via the Restriction Enzyme Recognition Site glyph) can persist in a SBOL Visual 2 diagram and still be considered compliant with SBOL Visual 2, where it is now classified as a Biopolymer Location (which is a superclass of cleavage sites). Thus, the Biopolymer Location glyph from SBOL Visual 2.0 is backwards compatible with the Restriction Enzyme Recognition Site glyph from SBOL Visual 1.0.

The 5' Sticky Restriction Site, 3' Sticky Restriction Site, and Blunt Restriction Site glyphs remain unchanged, and are more specific children/derivatives of the DNA-Stem Cleavage-Top glyph. 


\section{Composite}

\section{Associated SO term(s)}

Composite does not have an associated SO term, as it merely links a base glyph (with its own SO term) to a sub-diagram (comprising glyphs with their own associated SO terms).

\section{Recommended Glyph and Alternates}

The glyph for Composite is dashed "expanding lines" connecting any "base" glyph representing the more abstract composite (e.g., Omitted Detail, or Terminator, or Promoter) to a backbone diagramming the contents of the composite. Note the bounding box is indicating the location of the base glyph, and would scale with that glyph.

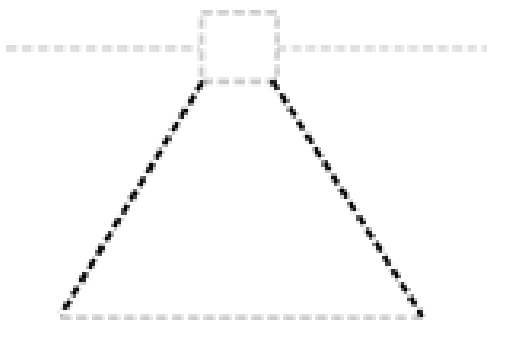

\section{Prototypical Example}

An "expression cassette" containing a ribosome entry site, coding sequence, and terminator. In this case, the recommended "base" glyph would be Engineered Region.

\section{Notes}

An "abbreviated" representation of composite, simply indicating that more structure is available, can be made by using short lines and placing only an Omitted Detail glyph in the secondary backbone. For example, here is an example of an abbreviated composite promoter:

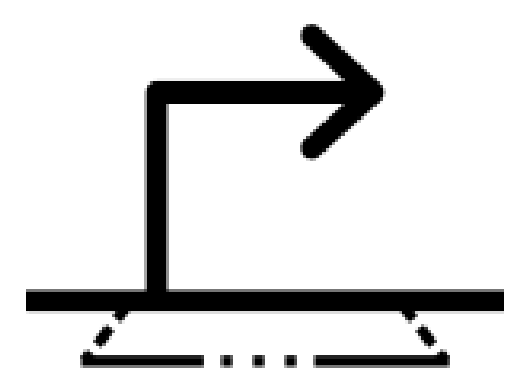

and a composite with an Engineered Region of otherwise unspecified content: 


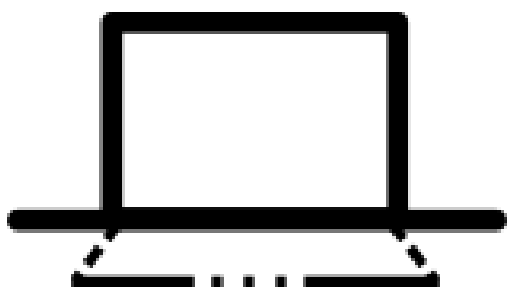




\section{Engineered Region}

\section{Associated SO term(s)}

SO:0000804 (Engineered Region)

\section{Recommended Glyph and Alternates}

Engineered Region is represented by a plain rectangle suggesting a blank slate to be written upon:

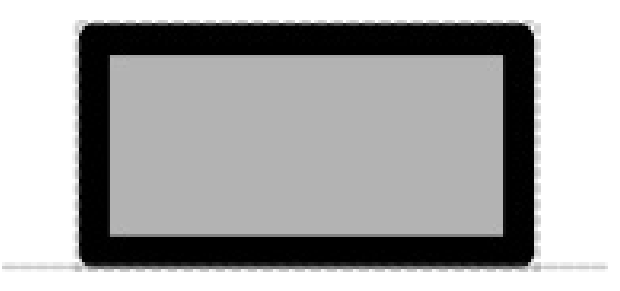

\section{Prototypical Example}

An "expression cassette" containing a ribosome entry site, coding sequence, and terminator.

\section{Notes}

this section deliberately blank 


\section{5' Overhang Site}

\section{Associated SO term(s)}

SO:0001932: 5' Overhang Site

SO:0001933: 3' Overhang Site

\section{Recommended Glyph and Alternates}

The $5^{\prime}$ overhang site glyph is an image of a strand of DNA extended on the $5^{\prime}$ edge of its forward strand:

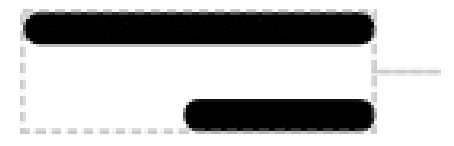

With a double-stranded backbone:

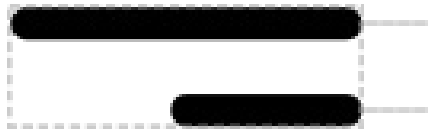

\section{Prototypical Example}

EcoRI site after cleavage.

\section{Notes}

The complementary 3' Overhang Site glyph is a reflection of the 5' Overhang Site. 


\section{5' Sticky Restriction Site}

\section{Associated SO term(s)}

SO:0001975 (5' Sticky Restriction Site)

SO:0001976 (3' Sticky Restriction Site)

\section{Recommended Glyph and Alternates}

The $5^{\prime}$ sticky restriction site glyph is an image of the lines along which two strands of DNA will be cut into 5 ' sticky ends. Vertical position with respect to the backbone is in a break in a single backbone:

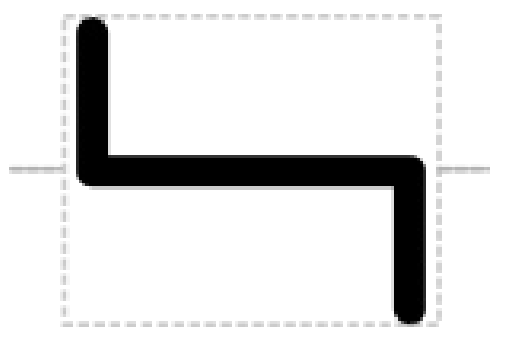

and between strands of a double backbone:

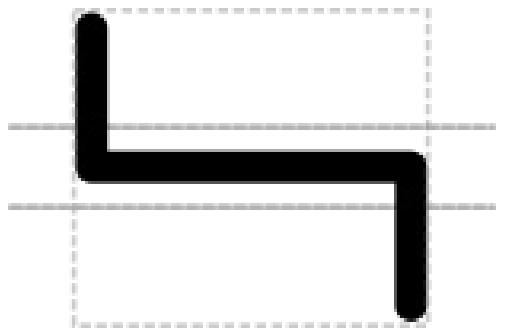

\section{Prototypical Example}

EcoRI restriction site.

\section{Notes}

The complementary 3' Sticky Restriction Site glyph is a reflection of the 5' Sticky Restriction Site. 


\section{Insulator}

\section{Associated SO term(s)}

SO:0000627

\section{Recommended Glyph and Alternates}

The insulator glyph is a box inside another box that isolates it from its environment:

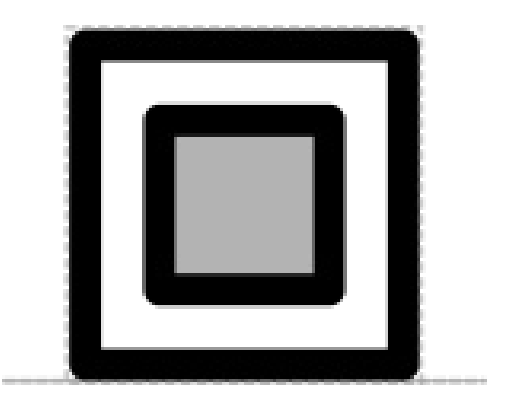

\section{Prototypical Example}

RiboJ

Notes

this section deliberately blank 


\section{Biopolymer Location}

\section{Associated SO term(s)}

SO:0000699 (Junction, Boundary, Breakpoint)

SO:0001236 (Base)

SO:0001237 (Amino Acid)

\section{Recommended Glyph and Alternates}

Biopolymer Location is a "stem-top" glyph for describing small sites. In this system:

- the top glyph indicates the type of site (e.g., Biopolymer Location)

- the stem glyph indicates whether the site affects DNA, RNA, or protein (respectively: straight, wavy, or looped)

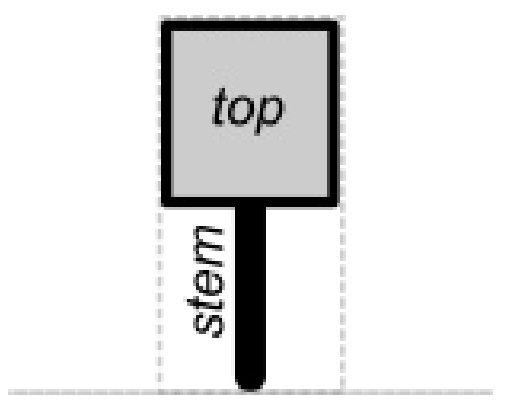

The RECOMMENDED top for Biopolymer Location is a circle, reminiscent of a pin stuck into a location (in order: DNA, RNA, Protein): 


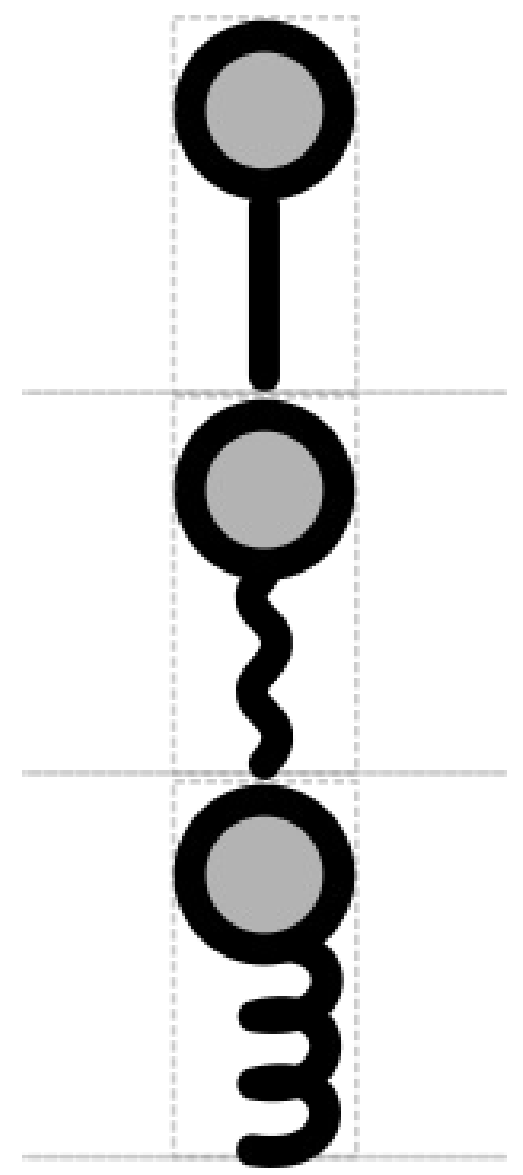

An alternative is to have "nothing" for the top, just an extended version of the stem itself (in order: DNA, RNA, Protein):

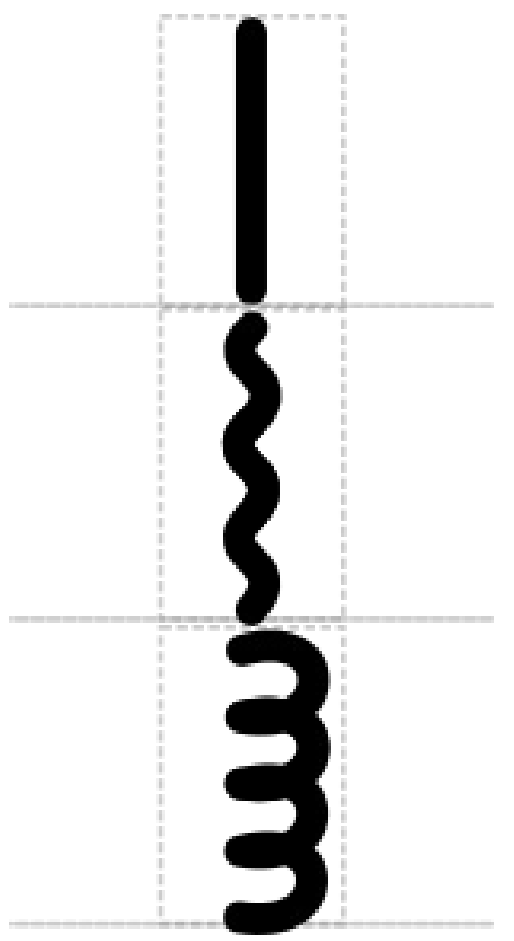




\section{Prototypical Example}

CRISPR-targeted insertion site, protease site, mutation site

\section{Notes}

Biopolymer Location is a general glyph for all zero- and one-length sequence features, including insertion and deletion sites and $\mathrm{X}$-ase cut sites.

Note also that Biopolymer Location does not cover stability elements, since their length is typically multiple bases / amino acids.

Describing a Restriction Enzyme Cleavage Site with a vertical line glyph on a DNA backbone (as done previously in SBOL Visual 1.0 via the Restriction Enzyme Recognition Site glyph) can persist in a SBOL Visual 2 diagram and still be considered compliant with SBOL Visual 2, where it is now classified as a Biopolymer Location (which is a superclass of cleavage sites). Thus, the Biopolymer Location glyph from SBOL Visual 2.0 is backwards compatible with the Restriction Enzyme Recognition Site glyph from SBOL Visual 1.0. 


\section{No Glyph Assigned}

\section{Associated SO term(s)}

Any SO term that is not covered by any glyph besides the root Sequence Feature

\section{Recommended Glyph and Alternates}

When a part has no assigned glyph it is RECOMMENDED that a user provide their own glyph. The user is also encouraged to submit the new glyph for possible adoption into the SBOLv standard. An alternative is brackets, suggesting information that needs to be filled in:

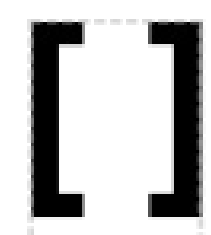

As a best practice, it is RECOMMENDED that the name of the term be put in between the brackets.

\section{Prototypical Example}

No Glyph Assigned is intended to be used for any Component that is not covered by other SBOL Visual glyphs.

For example, at present there is no glyph recommended for representing a transposon.

\section{Notes}

No Glyph Assigned is intended for constructs with a defined specific role that happens to not yet be covered by available approved glyphs (other than the root "Sequence Feature"). It is more likely to appear in machine-generated diagrams than in human-generated diagrams, since humans are likely to invent and use their own glyph for the purpose. 


\section{Non-Coding RNA Gene}

\section{Associated SO term(s)}

SO:0001263: Non-Coding RNA Gene

SO:0000834: Mature Transcript Region

\section{Recommended Glyph and Alternates}

The non-coding RNA glyph is a rectangular box whose top is a single-stranded RNA "wiggle":

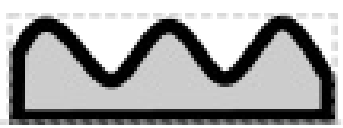

\section{Prototypical Example}

gRNA sequence for targeting a dCas9 repressor

\section{Notes}

This section left deliverately blank 


\section{Omitted Detail}

\section{Associated SO term(s)}

No SO term is associated with Omitted Detail, as it is indicating that something is not being represented.

\section{Recommended Glyph and Alternates}

The Omitted Detail glyph is a break in the backbone with an ellipsis to indicate that material would normally be in that location:

\section{Prototypical Example}

A diagram in which a sequence feature is not drawn.

\section{Notes}

This glyph actually places a "break" in the nucleic acid backbone. 


\section{Operator / Binding Site}

\section{Associated SO term(s)}

SO:0000057 Operator

SO:0000409 Binding Site

\section{Recommended Glyph and Alternates}

The operator glyph is an open "cup" suggesting a binding location:

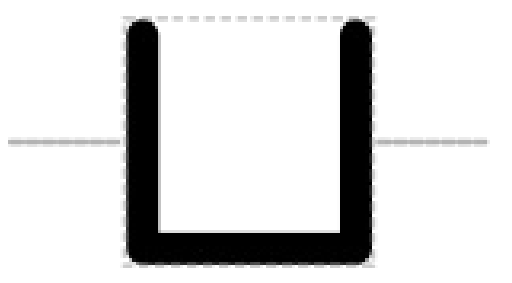

\section{Prototypical Example}

Gal4 binding site in an activatable promoter.

\section{Notes}

This glyph puts a "dent" in the backbone line. 


\section{Origin of Replication}

\section{Associated SO term(s)}

SO:0000296

\section{Recommended Glyph and Alternates}

The origin of replication glyph is a circle suggesting the "bulge" opened in a piece of circular DNA when replication is beginning:

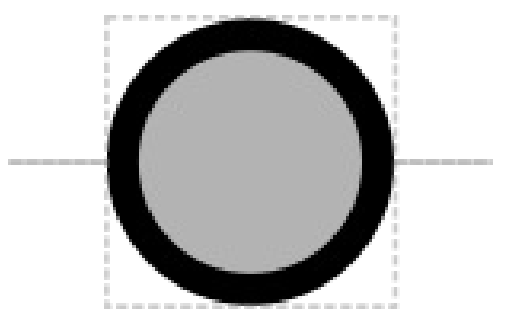

\section{Prototypical Example}

human herpesvirus-6 OOR

\section{Notes}

The label on an origin of replication glyph is RECOMMENDED as the location to label either specifically the identity of the origin of replication or the name of the entire plasmid backbone more generally. 


\section{Origin of Transfer}

\section{Associated SO term(s)}

SO:0000724: Origin of Transfer

\section{Recommended Glyph and Alternates}

The origin of transfer glyph is circular like origin of replication, but also includes an outbound arrow:

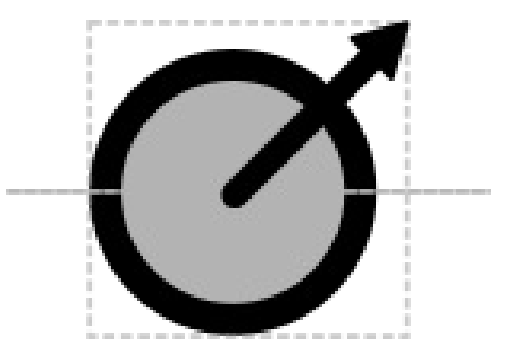

\section{Prototypical Example}

oriT

\section{Notes}

This section left deliberately blank 


\section{PolyA Site}

\section{Associated SO term(s)}

SO:0000553: polyA Site

\section{Recommended Glyph and Alternates}

The polyA site glyph is a sequence of As sitting atop the backbone:

\section{AAA}

\section{Prototypical Example}

polyA tail on mammalian coding sequence

\section{Notes}

This section left deliberately blank 


\section{Primer Binding Site}

\section{Associated SO term(s)}

SO:0005850

\section{Recommended Glyph and Alternates}

The primer binding site glyph is a line with a bent end suggesting a partially complementary strand of nucleic acid attaching to the backbone:

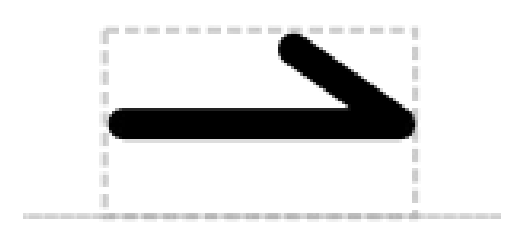

\section{Prototypical Example}

seq-F

\section{Notes}

this section deliberately blank 


\section{Promoter Site}

\section{Associated SO term(s)}

SO:0000167

\section{Recommended Glyph and Alternates}

The promoter glyph is a bent arrow pointing forward, suggesting the action of transcription from its transcription start site:

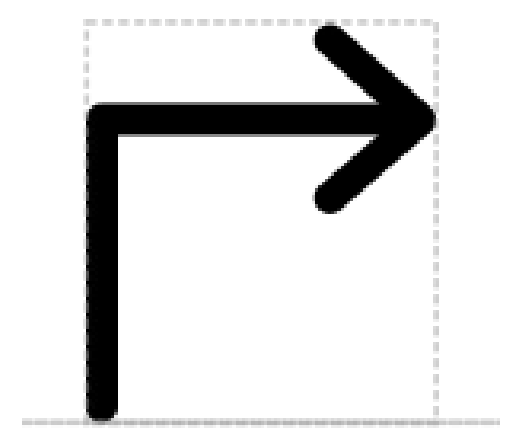

\section{Prototypical Example}

The lacYZA promoter

\section{Notes}

this section deliberately blank 


\section{Ribosome Entry Site}

\section{Associated SO term(s)}

SO:0000139: Ribosome Entry Site

\section{Recommended Glyph and Alternates}

The ribosome entry promoter glyph is a half-ovoid sitting on the backbone, suggesting an attached ribosome beginning transcription:

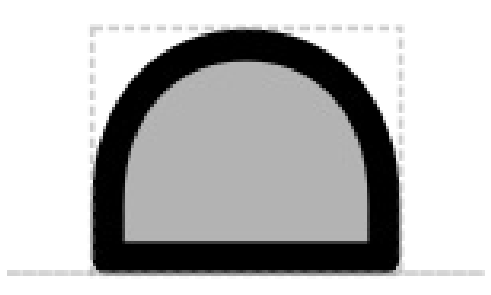

\section{Prototypical Example}

T7g10 ribosome binding site

\section{Notes}

this section deliberately blank 


\section{Signature}

\section{Associated SO term(s)}

SO:0001978

\section{Recommended Glyph and Alternates}

The signature glyph is a box sitting atop the backbone with an $\mathrm{X}$ and line inside it, suggesting a signature on a form:

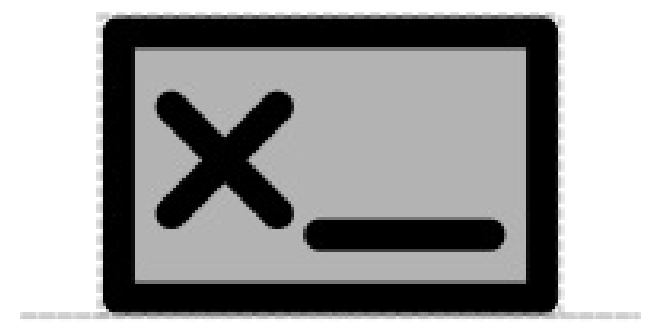

\section{Prototypical Example}

DNA Barcode

\section{Notes}

this section deliberately blank 


\section{Specific Recombination Site}

\section{Associated SO term(s)}

SO:0000299: Specific Recombination Site

\section{Recommended Glyph and Alternates}

The specific recombination site glyph is a triangle, centered on the backbone, as has appeared in a number of recombinase circuit papers:

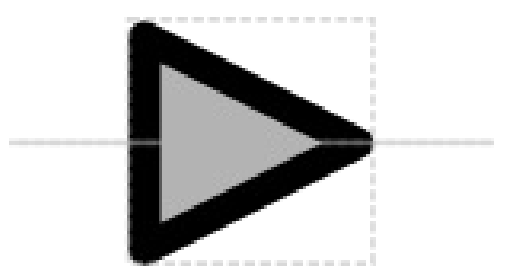

\section{Prototypical Example}

flippase recognition target (FRT) site

\section{Notes}

This section left deliberately blank 


\section{Stability Element}

\section{Associated SO term(s)}

SO:0001955, SO:0001546 (Protein Stability Element)

SO:0001979 (RNA Stability Element)

No SO term is currently associated with DNA stability.

\section{Recommended Glyph and Alternates}

Stability Element is a "stem-top" glyph for describing small sites. In this system:

- the top glyph indicates the type of site (e.g., Stability Element)

- the stem glyph indicates whether the site affects DNA, RNA, or protein (respectively: straight, wavy, or looped)

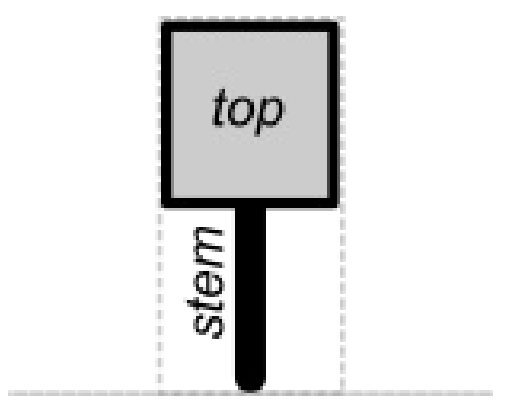

The top for a Stability Element is a pentagon suggesting the shape of a shield, on top of a stem connecting to the backbone at the point where the stability element is located (in order: DNA, RNA, Protein): 


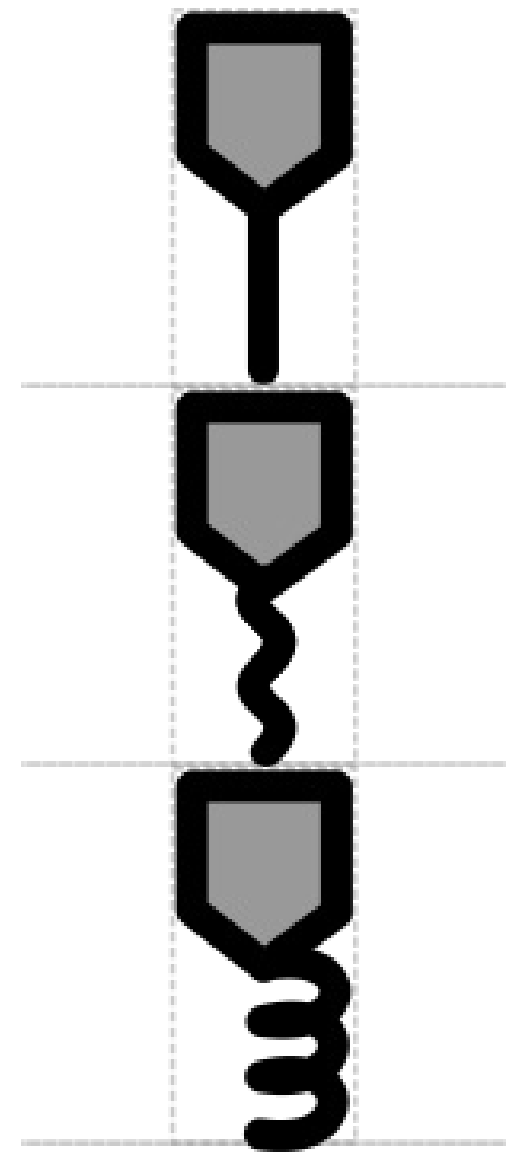

\section{Prototypical Example}

PEST tag, 3' Hairpin

\section{Notes}

RNA Stability Element glyph was previously also associated with SO:0001957, but that SO term has been declared obsolete in Sequence Ontology.

This glyph is not backwards compatible with SBOL Visual 1.0.

Despite both being stem-top glyphs, Biopolymer Location is not a parent to Stability Element, since the length of a Stability Element is typically multiple bases / amino acids. 


\section{Stop Site (Transcrition/Translation End Point)}

\section{Associated SO term(s)}

SO:0000616 Transcription End Site

SO:0000319 Stop Codon;

SO:0000327 Coding End, Translation Termination Site, Translation End

\section{Recommended Glyph and Alternates}

Transcription/Translation End Point is a "stem-top" glyph for describing small sites. In this system:

- the top glyph indicates the type of site (e.g., Biopolymer Location)

- the stem glyph indicates whether the site affects DNA, RNA, or protein (respectively: straight, wavy, or looped)

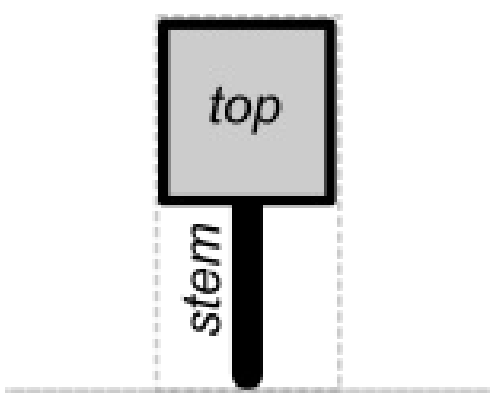

The Transcription/Translation End Point top is an asterisk in a circle (in order: transcription, translation):

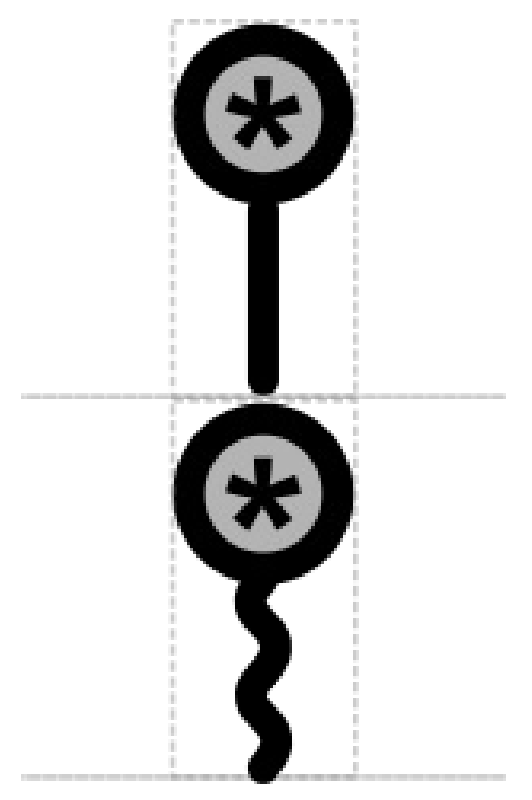

\section{Prototypical Example}


Location where a terminator causes transcription to stop, stop codon

\section{Notes}

Note that the number of points in the asterisk is not specified, accomodating font differences. 


\section{Terminator}

\section{Associated SO term(s)}

SO:0000141: Terminator

\section{Recommended Glyph and Alternates}

The terminator is a T sitting atop the backbone:

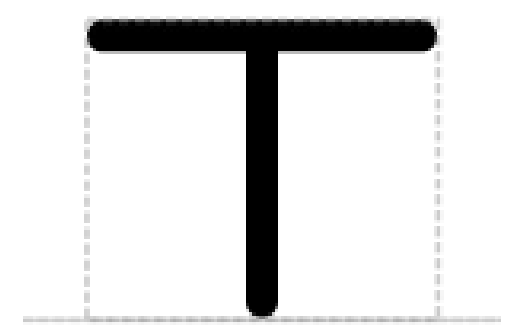

\section{Prototypical Example}

T1 terminator

\section{Notes}

this section deliberately blank 


\section{Unspecified}

\section{Associated SO term(s)}

Unspecified: SO:0000110 Sequence Feature

\section{Recommended Glyph and Alternates}

Unspecified is represented by the unicode "replacement character" glyph, indicating a missing or invalid symbol, is RECOMMENDED:

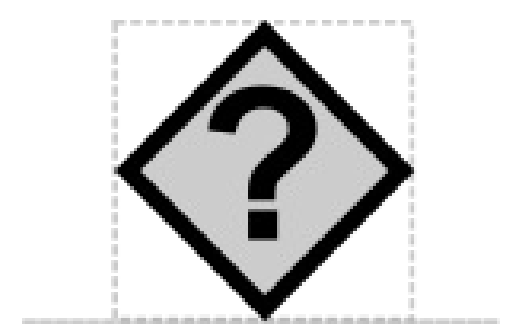

A half-rounded rectangle, the SBGN glyph for a nucleic acid, is an alternative:

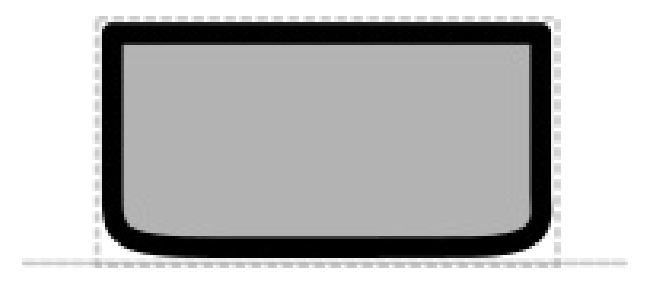

\section{Prototypical Example}

An anonymous sequence that is missing any information about its nature or intended purpose.

\section{Notes}

The Unspecified glyph is intended for showing where a sequence's role is missing (or, equivalently, given only the uninformative "Sequence Feature" root role). It should never appear with wellcurated designs or diagrams. 


\section{A.2 Molecular Species Glyphs}

These glyphs represent molecular species in a diagram, and include a bounding box (grey dashed box) but are not connected to any nucleic acid backbone. 


\section{Complex}

\section{Associated BioPAX term(s)}

Complex: http://www.biopax.org/release/biopax-level3.owl\#Complex

\section{Recommended Glyph and Alternates}

The RECOMMENDED glyph for a complex is a composite of the glyphs for the molecules of comprising the complex. For example, a protein bound to a small molecule, a guide RNA, or another protein:

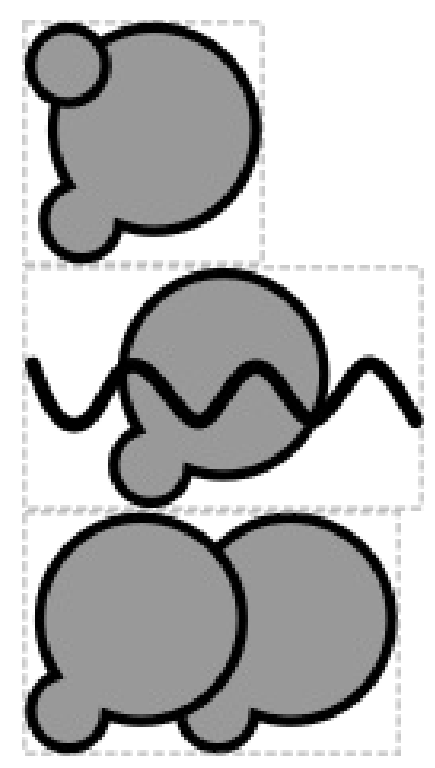

An alternative is the SBGN "cornered rectangle" glyph for a complex:

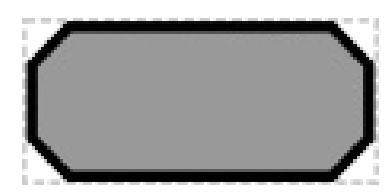

\section{Prototypical Example}

Arabinose bound to AraC

\section{Notes}

This section left intentionally blank 


\section{Double-Stranded Nucleic Acid}

\section{Associated BioPAX term(s)}

Dna: http://www.biopax.org/release/biopax-level3.owl\#Dna

\section{Recommended Glyph and Alternates}

The RECOMMENDED glyph for dsNA is a double-helix:

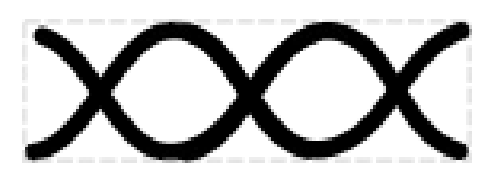

An alternative is the SBGN "nucleic acid" half-round rectangle:

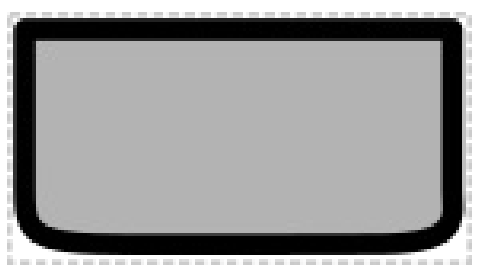

\section{Prototypical Example}

DNA fragment during assembly

\section{Notes}

This section left intentionally blank 


\section{Macromolecule}

\section{Associated BioPAX term(s)}

Protein: http://www.biopax.org/release/biopax-level3.owl\#Protein

\section{Recommended Glyph and Alternates}

The macromolecule glyph is a diagonally offset union of a large and small circle, intended to invoke the complex shapes of proteins:

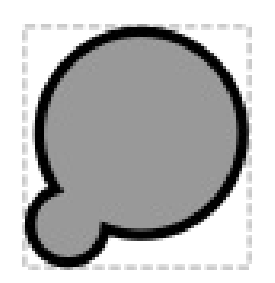

An alternative is the SBGN macromolecule glyph, a rounded rectangle:

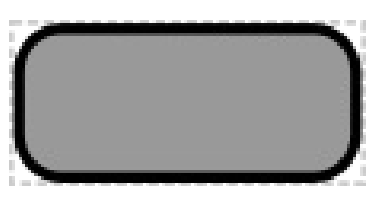

\section{Prototypical Example}

AraC

\section{Notes}

It is unclear whether this should be just "Protein" or whether we also want it to be able to repesent multi-component elements like a protein composed of multiple sub-units or a complex polymer. 


\section{No Glyph Assigned}

\section{Associated BioPAX term(s)}

Any BioPAX type that is not covered by any glyph besides the root

\section{Recommended Glyph and Alternates}

When a species has no assigned glyph it is RECOMMENDED that a user provide their own glyph. The user is also encouraged to submit the new glyph for possible adoption into the SBOLv standard.

An alternative option is to have a bracket, suggesting information that needs to be filled in:

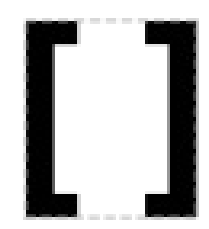

\section{Prototypical Example}

No Glyph Assigned is intended to be used for any chemical species whose type is not covered by other SBOL Visual glyphs.

\section{Notes}

No Glyph Assigned is intended for molecular species with a defined specific type that happens to not yet be covered by available approved glyphs (other than the root). It is more likely to appear in machine-generated diagrams than in human-generated diagrams, since humans are likely to invent and use their own glyph for the purpose. 


\section{Small Molecule}

\section{Associated BioPAX term(s)}

Small Molecule: http://www.biopax.org/release/biopax-level3.owl\#SmallMolecule

\section{Recommended Glyph and Alternates}

The small molecule glyph is a circle that stretches sideways into a "stadium" to accomodate longer names:

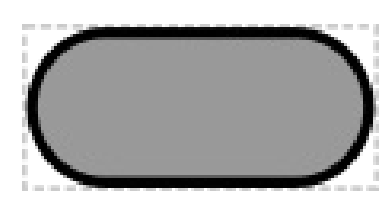

\section{Prototypical Example}

Arabinose

\section{Notes}

This section left intentionally blank 


\section{Single-Stranded Nucleic Acid}

\section{Associated BioPAX term(s)}

Rna: http://www.biopax.org/release/biopax-level3.owl\#Rna

\section{Recommended Glyph and Alternates}

The RECOMMENDED glyph for ssNA is a wiggly line:

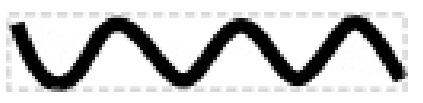

An alternative is the SBGN "nucleic acid" half-round rectangle:

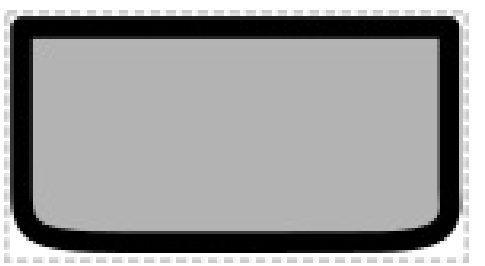

\section{Prototypical Example}

mRNA, gRNA, siRNA

\section{Notes}

This section left intentionally blank 


\section{Unspecified}

\section{Associated BioPAX term(s)}

PhysicalEntity: http://www.biopax.org/release/biopax-level3.owl\#PhysicalEntity

\section{Recommended Glyph and Alternates}

Unspecified is RECOMMENDED to be represented by the unicode "replacement character" glyph, indicating a missing or invalid symbol:

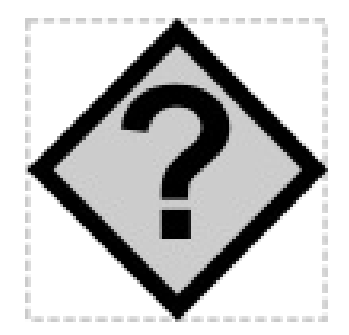

An alternative is the SBGN "generic species" glyph, which is an ellipse:

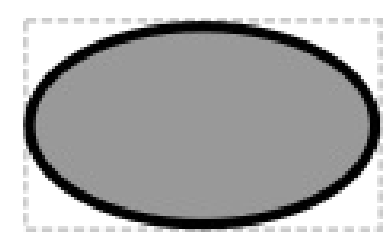

\section{Prototypical Example}

An anonymous chemical species that is missing any information about its nature or intended purpose.

\section{Notes}

The Unspecified glyph is intended for showing where a chemical species' type is missing (or, equivalently, given only the uninformative root role). It should never appear with well-curated designs or diagrams. 


\section{A.3 Interaction Glyphs}

These glyphs are different forms of "arrow" representing interactions between sequence features and/or molecular species. As arrows, they are extensible and do not have a separately identified bounding box. 


\section{Control}

\section{Associated SBO term(s)}

SBO:0000168 Control

\section{Recommended Glyph and Alternates}

An arrow with a diamond head:

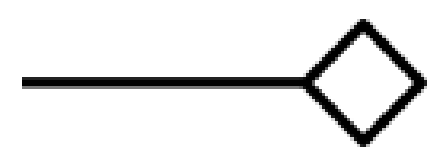

\section{Prototypical Example}

Inversion of a sequence flanked by FRT sites by FLP recombinase

Notes

This section left intentionally blank 


\section{Degradation}

\section{Associated SBO term(s)}

SBO:0000179 Degradation

\section{Recommended Glyph and Alternates}

Identical to the Process glyph, but with an empty set at the sink of the arrowhead:

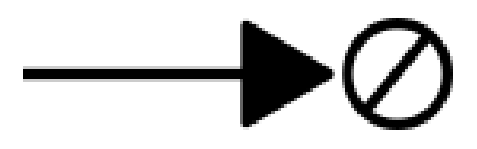

\section{Prototypical Example}

Cellular recycling of mRNA

\section{Notes}

This section left intentionally blank 


\section{Inhibition}

\section{Associated SBO term(s)}

SBO:0000169 Inhibition

\section{Recommended Glyph and Alternates}

An arrow whose head is a bar, suggesting blocking:

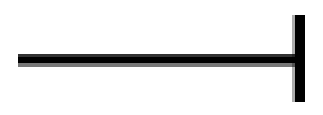

\section{Prototypical Example}

Repression of pTAL14 promoter by TAL14

\section{Notes}

This section left intentionally blank 


\section{Process}

\section{Associated SBO term(s)}

SBO:0000375 Process

\section{Recommended Glyph and Alternates}

An arrow with a filled head the same color as the line:

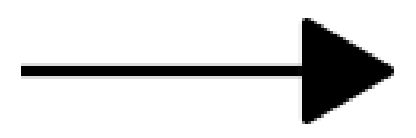

\section{Prototypical Example}

Production of Green Fluorescent Protein (GFP) from the gfp Coding Sequence

\section{Notes}

The assocated SBO term also covers:

- SBO:0000176 Biochemical Reaction

- SBO:0000589 Genetic Production (source is DNAcomponent, sink is usually RNA or Macromolecule)

- SBO:0000177 Non-covalent Binding (sink is a Complex) 


\section{Stimulation}

\section{Associated SBO term(s)}

SBO:0000170 Stimulation

\section{Recommended Glyph and Alternates}

An arrow with an head that is empty or of a different color than the line:

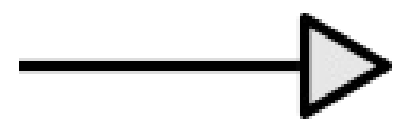

\section{Prototypical Example}

Activation of pTAL14 promoter by Gal4VP16 activator

\section{Notes}

This section left intentionally blank 


\section{A.4 Interaction Node Glyphs}

These glyphs are placed at the junctions of edges to represent biochemical processes, and include a bounding box (grey dashed box) but are not connected to any nucleic acid backbone. Grey dashed lines provide examples of how edges may connect to the glyph. 


\section{Association}

\section{Associated SBO term(s)}

SBO:0000177 Non-Covalent Binding

\section{Recommended Glyph and Alternates}

A circular node:

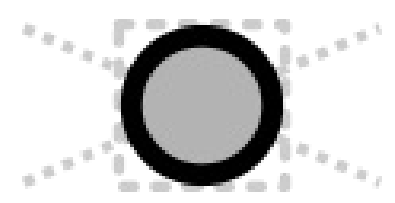

\section{Prototypical Example}

Association of gRNA and Cas9 to form an active CRISPR complex.

\section{Notes}

The association glyph is based on the SBGN Process Description association glyph. 


\section{Dissociation}

\section{Associated SBO term(s)}

SBO:0000180 Dissociation

\section{Recommended Glyph and Alternates}

An circular node inside another circle

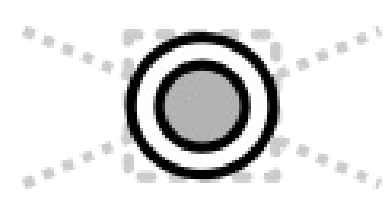

\section{Prototypical Example}

Dissociation of an active CRISPR complex into gRNA and Cas9.

\section{Notes}

The dissociation glyph is based on the SBGN Process Description dissociation glyph. 


\section{Process}

\section{Associated SBO term(s)}

SBO:0000375 Process

\section{Recommended Glyph and Alternates}

A square node:

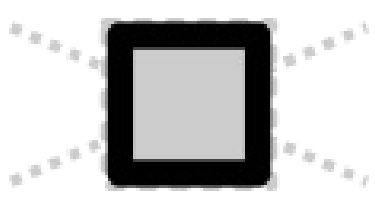

\section{Prototypical Example}

Association of gRNA and Cas9 to form an active CRISPR complex.

\section{Notes}

The process glyph is based on the SBGN Process Description process glyph.

The assocated SBO term also covers:

- SBO:0000176 Biochemical Reaction

- SBO:0000177 Non-covalent Binding (sink is a Complex) 


\section{B Examples}

This section contains prototypical examples, including use of all current glyphs to attempt to ensure that their use is clear.

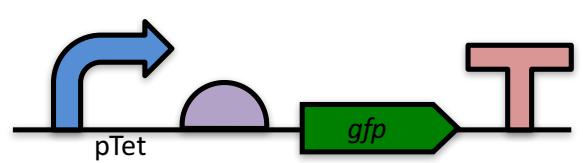

Figure 24: DNA sequence for a functional unit in which the pTet promoter and an anonymous ribosome entry site regulate expression of a coding sequence for GFP, ended by a terminator.

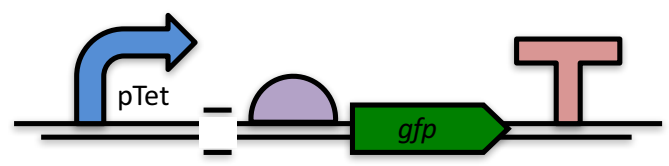

Figure 25: The same functional unit as in Figure 24, with additional assembly-focused information: there is a 5' overhang before the promoter, a 3' overhand after the terminator, and an assembly scar between the promoter and the ribosome entry site left over from a prior step of assembly.

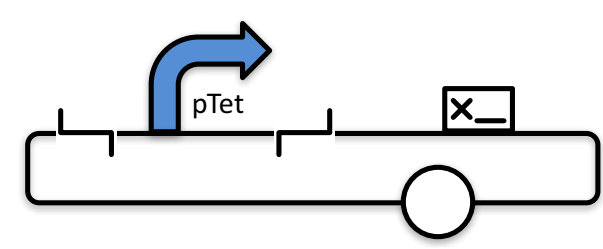

Figure 26: Promoter pTet stored in a circular plasmid. The promoter is prepared for being cut out of the plasmid: it is preceded by a 5' sticky end restriction site and followed by a 3' stick end restriction site. In addition, the plasmid has been bar-coded with a signature and has its origin of replication marked.

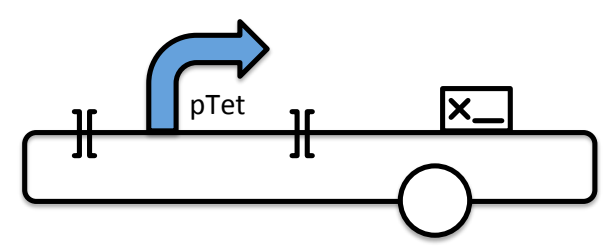

Figure 27: Promoter stored in a plasmid as in Figure 26, except that the restriction sites before and after the promoter are blunt-end. 


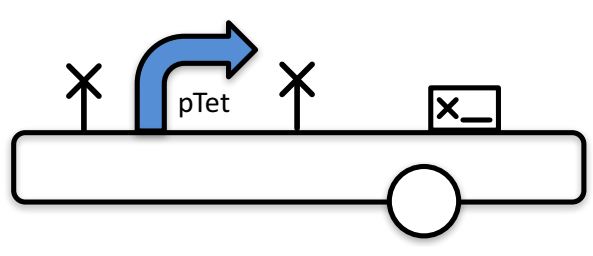

Figure 28: Promoter stored in a plasmid as in Figure 26, except that the cut structure of the restriction sites before and after the promoter is not specified.

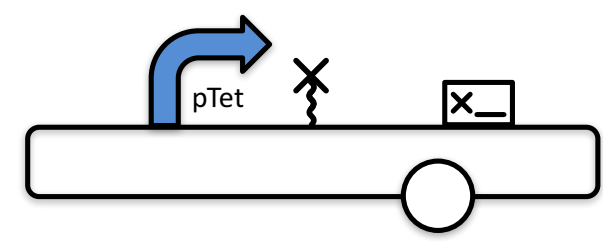

Figure 29: Promoter stored in a plasmid as in Figure 26, except that there is a ribonuclease site after the promoter rather than restriction sites flanking it.

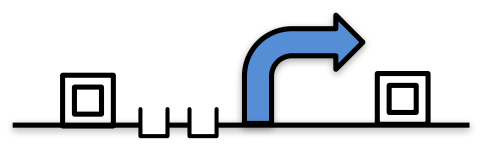

Figure 30: Detailed design of a promoter, in which the transcription start site is preceded by two operator sites where regulators bind, and the whole is flanked by insulators.

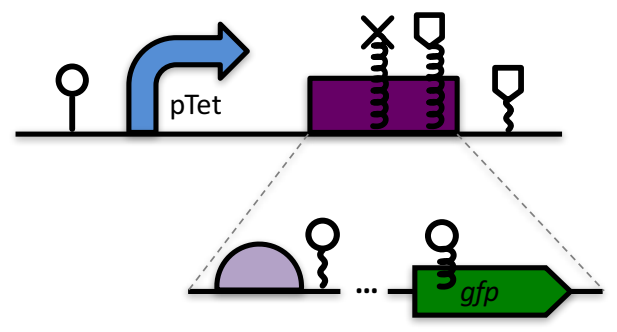

Figure 31: Promoter regulating the production of an engineered composite sequence that includes RNA and protein stability elements at its 3 ' end, as well as an internal site for protease cleavage, as well as the expansion of the composite to show it contains a ribosome entry site. coding sequence, and other omitted details. Single residue locations of interest are indicated for the DNA (before the promoter), RNA (after the ribosome entry site), and protein (in the CDS).

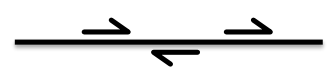

Figure 32: DNA sequence with three primer binding sites.

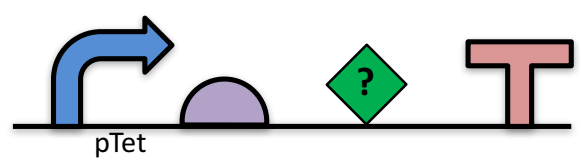

Figure 33: The same functional unit as in Figure 24, except that information about the CDS is missing, leaving it to fall back on the default unspecified glyph. 


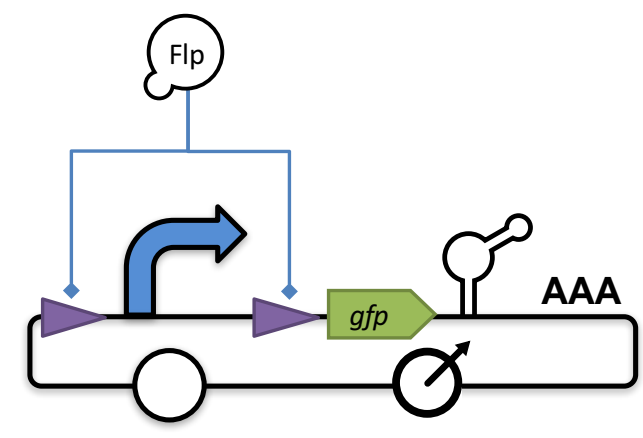

Figure 34: Promoter regulating the expression of GFP, which is also regulated by an aptamer between it and the poly-A tail of the transcript. The promoter can be cut out by a pair of recombinase target sites, which are acted on by the Flp protein. The whole construct is stored in a circular plasmid with an origin of replication and also an origin of transfer.

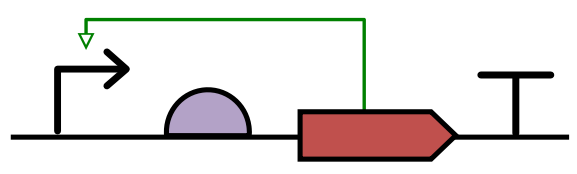

Figure 35: Promoter stimulated by the CDS that it regulates.

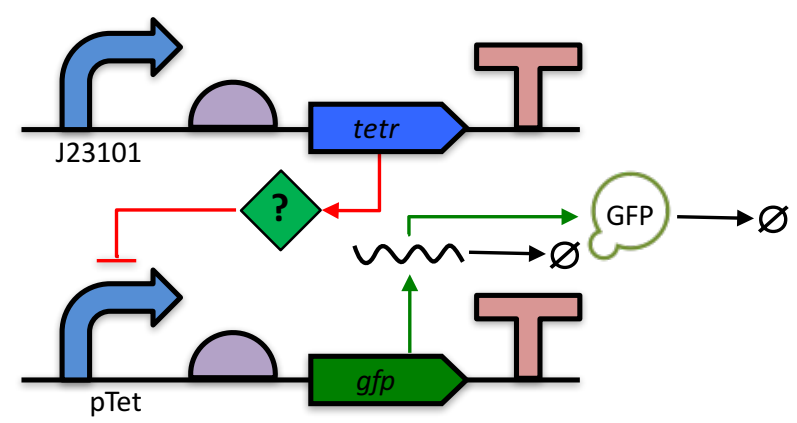

Figure 36: Constitutive production of TetR, except that information about the protein is missing, leaving it as the default unspecified glyph. TetR represses the pTet promoter, which is regulating production of GFP. The diagram of GFP production explicitly includes the intermediate mRNA and the degradation of both the mRNA and protein products.

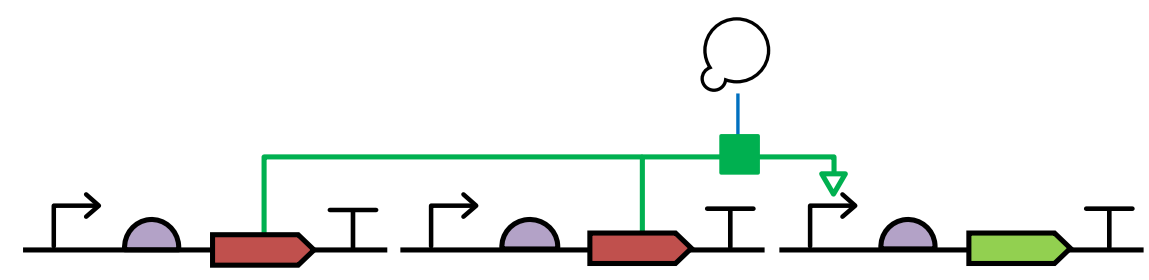

Figure 37: Phosphorylation of an inactive transcription factor (produced by two different CDSs) by a kinase to form an active transcriptional activator, which then stimulates a promoter. 


\section{Relationship to SBOL Visual 1.0}

SBOL Visual 2.0 differs from SBOL Visual 1.0 in the following major ways:

- Diagram syntax is expanded to include functional interactions and other molecular species.

- The relationship between diagrams and the SBOL data model is made explicit.

- A number of requirements and best practices are specified for glyphs and diagrams, including:

- Glyphs include information on interior, bounding box, and recommended backbone alignment.

- Sequence feature glyphs are required to have their bounding boxes contact the nucleic acid backbone.

- Nucleic acid diagrams now require the nucleic acid backbone line, and the number of lines allowed in various circumstances is constrained.

- Explicit statement of when a glyph can and cannot be used to represent a particular element of a diagram.

- Labels that name objects are distinguished from other types of textual annotation.

- Explicit statement of which aspects of a symbol are not controlled.

- Symbol variants are now supported.

In addition, the collection of sequence feature glyphs have been expanded and modified in the following ways:

- All non-ambiguous glyphs have been provided with bounding box, interior, and recommended backbone alignment.

- The User Defined glyph has been split into Unspecified, No Glyph Assigned, Engineered Region, and Composite.

- Glyphs have been added for Aptamer, Omitted Detail, Biopolymer Location, Non-Coding RNA Gene, Origin of Transfer, PolyA Site, and Specific Recombination Site.

- The following ontology terms have been assigned or adjusted:

- Ribonuclease Site has been assigned SO:0001977.

- 5' Sticky End Restriction Site has been assigned SO:0001975.

- 3' Sticky End Restriction Site has been assigned SO:0001976.

- Signature has been assigned SO:0001978.

- RNA Stability Element has been updated from the obsolete SO:0001957 to the current SO:0001979

- Restriction Enzyme Recognition Site, in addition to SO:0000139 has a second definition as SO:0000061.

- 5' Overhang Site and 3' Overhang Site were erroneously listed with their ontology terms exchanged; this has been fixed. 


\section{References}

Courtot, M., Juty, N., Knüpfer, C., Waltemath, D., Zhukova, A., Dräger, A., Dumontier, M., Finney, A., Golebiewski, M., Hastings, J., et al. (2011). Controlled vocabularies and semantics in systems biology. Molecular systems biology, $7(1): 543$.

Eilbeck, K., Lewis, S. E., Mungall, C. J., Yandell, M., Stein, L., Durbin, R., and Ashburner, M. (2005). The Sequence Ontology: a tool for the unification of genome annotations. Genome biology, 6(5):R44.

Goldberg, R. N., Cary, M., and Demir, E. (2010). BioPAX: A community standard for pathway data sharing. Nature Biotechnology, 28(9).

Le Novère, N., Hucka, M., Mi, H., Moodie, S., Schreiber, F., Sorokin, A., Demir, E., Wegner, K., Aladjem, M. I., Wimalaratne, S. M., Bergman, F. T., Gauges, R., Ghazal, P., Kawaji, H., Li, L., Matsuoka, Y., Villéger, A., Boyd, S. E., Calzone, L., Courtot, M., Dogrusoz, U., Freeman, T. C., Funahashi, A., Ghosh, S., Jouraku, A., Kim, S., Kolpakov, F., Luna, A., Sahle, S., Schmidt, E., Watterson, S., Wu, G., Goryanin, I., Kell, D. B., Sander, C., Sauro, H., Snoep, J. L., Kohn, K., and Kitano, H. (2009). The systems biology graphical notation. Nat. Biotechnol., 27(8):735-741. 